Canadian

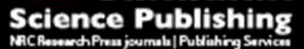

Canadian Journal of Civil Engineering Revue canadienne de génie civil

\title{
Analysis of Volumetric Properties of Bituminous Mixtures Using Cellular Phones and Image Processing Techniques
}

\begin{tabular}{|r|l|}
\hline Journal: & Canadian Journal of Civil Engineering \\
\hline Manuscript ID & cjce-2017-0085.R1 \\
\hline Manuscript Type: & Article \\
\hline Date Submitted by the Author: & 30 -May-2017 \\
\hline Complete List of Authors: & $\begin{array}{l}\text { Obaidat, Mohammed; Jordan University of Science and Technology Faculty } \\
\text { of Engineering, Civil Engineering Department } \\
\text { Ghuzlan, Khalid; Jordan University of Science and Technology Faculty of } \\
\text { Engineering, Civil Engineering Department } \\
\text { Alawneh, Mai; Jordan University of Science and Technology Faculty of } \\
\text { Engineering, Civil Engineering Department }\end{array}$ \\
\hline $\begin{array}{r}\text { Is the invited manuscript for } \\
\text { consideration in a Special } \\
\text { Issue? : }\end{array}$ & N/A \\
\hline Keyword: & $\begin{array}{l}\text { Cellular Phone Images (CPI), ImageJ software, Volumetric Properties, VMA, } \\
\text { VFA }\end{array}$ \\
\hline \multicolumn{2}{|c}{} \\
\hline
\end{tabular}




\section{Analysis of Volumetric Properties of Bituminous Mixtures Using

\section{Mohammed T. Obaidat}

Professor of Civil Engineering Jordan University of Science and Technology P.O. Box 3030, Irbid, 22110 Jordan. Tel: 00962-2-720-1000 Ext. 22125

Email: mobaidat@just.edu.jo

Khalid A. Ghuzlan (corresponding author)

Associate Professor of Civil Engineering Jordan University of Science and Technology P.O. Box 3030, Irbid, 22110 Jordan Tel: 00962-2-720-1000 Ext. 22076

Email: kaghuzlan@just.edu.jo

\footnotetext{
Mai M. Alawneh

Research Assistant, Department of Civil Engineering Jordan University of Science and Technology P.O. Box 3030, Irbid, 22110 Jordan Tel: 00962-2-720-1000 Ext. 22125 Email: mmalawneh13@eng.just.edu.jo

Submitted for Publication at The Canadian Journal of Civil Engineering May $30^{\text {th }}, 2017$
} 


\section{Abstract}

44 This study aims to develop the micro-analysis of the bituminous mixtures using Cellular 45 Phone Images (CPI) and Image Processing Techniques (IPT). A new methodology and 46 scheme was developed for faster and accurate procedure to compute volumetric design 47 parameters; Voids in Mineral Aggregate (VMA), Voids in Total Mix (VTM) and Voids Filled with Asphalt (VFA) using CPI and IPT instead of the conventional methods. Five types of cellular phones with different high resolutions were used to analyze the horizontal cross section (face) of HMA slices. A cellular phone digital mapping frame for Micro-structure of

51 the bituminous mixture for data collection was designed and implemented. New models for computations of volumetric design perimeters (VMA, VTM and VFA) were developed. Results showed that the best cellular phone for micro-analysis of the bituminous mixture is type D, even though it doesn't have the highest resolution, and the best height of capturing the 55 images is $35 \mathrm{~cm}$.

56

57

58

61 2 3 64 5 6 67
Key words: Cellular Phone Images (CPI), VMA, VTM, VFA, Volumetric Properties, ImageJ software 


\section{Introduction}

70 Asphalt concrete is prepared by mixing graded aggregate (coarse and fine aggregate) with 71 asphalt cement content and then compact the mixture to get a specific percentage of air void level. The basic components of asphalt concrete are divided into a graded aggregate, binder, and air void. Fine aggregates of the graded aggregate are embedded in the matrix of asphalt binder. The physical and mechanical properties of asphalt concrete mixtures depend on the quantities and mechanical properties of the individual constituents. Aggregate shape, orientation, and gradation also play a very important role in the mixture performance (You et al. 2013).

The volumetric design of bituminous mixtures requires consideration of the percentage of air voids in the total mixture (VTM), voids in the mineral aggregate (VMA), and voids filled with asphalt (VFA).The percentage of air voids is used as the basis for selecting the

81 percentage of asphalt binder content $(\mathrm{AC} \%)$ that will be used in the mixture. Conventional 82 procedures used to quantify the volumetric design parameters (VMA, VTM, and VFA) 83 require a series of exhaustive analytical and laboratory steps which depend on different types of specific gravities of the bituminous mixes (Asphalt Institute 1988). However, most of the available models for HMA volumetric analysis were developed without experimental measurements of the microstructure distribution. This was due to the difficulties associated

87 with the quantitative analysis of the microstructure, which has prevented continuum modeling 88 from becoming a state-of-the-practice technique for HMA engineering applications (Wang et 89 al. 2001).

90 One of the most effective methods is the digital image processing and analysis technique.

91 Tashman and Wang (2011) summarized the recent advances in imaging technology and its 92 applications to the characterization of HMA microstructure including aggregate distribution, 
aggregate contact, aggregate shape properties, and air void distribution. The micro-structure of HMA is linked to the macroscopic behavior of the material within the framework of integrated modeling by microstructure tensors that are incorporated in the modeling formulations.

Bessa et al. (2012) used digital image processing (DIP) techniques to characterize the distribution of aggregates within the asphalt mixes. Results show that using DIP is easier, faster, and more precise than the results from the laboratory tests.

Vadood et al. (2014) introduced a simple and quick approach to determine aggregate gradation in the HMA. Image processing techniques and colour space system were used on cylindrical HMA samples. Results showed that aggregate gradation can be detected with high accuracy.

Obaidat et al. (1998) examined the effectiveness of using a semi-automated computer-vision system to quantify the percentage of voids in mineral aggregates (VMA \%) of bituminous mixtures. The system that was used in this study was a hybrid system which utilized a planimeter surveying instrument, and a digital image analysis scheme. Thirty-nine Marshall Specimens were prepared using two types of aggregate; limestone and gravel. The values of VMA\% were obtained by the ASTM conventional procedure and the computer-vision procedure. For computation of the VMA\% by using the computer-vision procedure a normal case photography with uniform scale images was used for mapping horizontal and vertical cross sections of Marshall Specimens. Spatial filters and image processing operations were used to detect the aggregate edges. The results showed slight differences between VMA\% computed using conventional and the computer-vision procedures. This study emphasized that the low-resolution image was a major factor in reducing the accuracy of the computed VMA\%. Researchers started to use image analysis techniques to characterize the distribution of air voids and assess the gyratory compaction efforts in AC mixtures based on X-ray CT 
118 images (Tashman et al. 2001 and Tashman et al. 2002). You and Buttlar (2004) said that it

119 was challenging to get accurate data for asphalt mixtures from images of the cutting faces for

120 Superpave mixture specimens. The researchers got data for the morphology and positions of

121 aggregate, sand mastic, and air void phases of asphalt concrete. Images of the microstructure

122 can be obtained from a flat-bed scanner, X-ray computed tomography (CT), or X-ray Micro-

123 fluorescence. Image processing techniques are then worked to acquire the aggregate, binder

124 and air void shape and orientation. You et al. (2013) developed a microstructure

125 characterization technique to map the multi- phase nature of asphalt concrete using X-ray

126 micro-fluorescence. This study examined the morphology and positions of aggregates, sand

127 mastic, and air void phases of asphalt concrete. Three different samples of mixtures with air

128 void levels of $4 \%, 7 \%$, and $10 \%$ were analyzed using an X-ray microscope. The cylindrical

129 asphalt samples were cut into slabs. These slabs were polished; then white zinc-oxide powder

130 was pressed into the voids. This powder helped to discern air voids from aggregate and

131 binder. The relative intensities of pixels in the elemental images were used to categorize

132 pixels in each image according to the binder, the air voids, and the aggregate using multi-

133 spectral analysis techniques. The images were analyzed and the aggregate gradation was

134 calculated and compared with the real gradation. It was concluded that the microstructure

135 characterization techniques via capturing the multi-phase nature of asphalt concrete using $\mathrm{x}$ -

136 ray micro-fluorescence were moderately successful.

137 Zelelew and Papagiannakis (2007) developed the Digital Image Processing (DIP) algorithm

138 called Volumetric-based Global Minima (VGM). It is a thresholding algorithm for processing

139 the asphalt concrete mixtures X-ray CT images. The image preprocessing and gray scale

140 thresholding were considered for characterizing of the asphalt concrete mixtures

141 microstructure X-ray CT images. There were briefed descriptions of different types of DIP

142 techniques applicable to characterize and simulate of asphalt concrete mixtures that include 
143 three-dimensional representation, edge detection, and segmentation of mastic and aggregate

144 objects. The method consists of Volumetric-driven thresholding based on a global minimum

145 percent error approach that utilizes thresholding criterion the actual volumetric properties of

146 the asphalt mixtures. It was applied to images of cross-sections of asphalt mixtures cores.

147 Generally, the VGM processed images are significantly improved compared to the raw X-ray

148 CT images.

149 The usage of image processing is growing fast in many aspects of science and engineering

150 application, in parallel with the distribution of cellular phones and their contribution in most

151 of the life issues. This is why this research can be considered as the first of its kind, since it

152 uses cellular phone images for micro-analysis of bituminous mixture by extracting the

153 volumetric design parameters (VMA, VTM, and VFA) from the cellular phone images. The

154 micro-structure of the bituminous mixture will affect the macro-structure of the pavement and

155 its performance. The conventional methods of the micro-analysis of bituminous mixture

156 (computation of the volumetric design parameters for Superpave mixtures and determination

157 of aggregate shape indices) require a series of exhaustive analytical and laboratory steps,

158 special technicians and they are considered to be time-consuming. The main limitation of X-

159 ray $\mathrm{CT}$ is that the equipment is expensive, and therefore difficult to be available for every

160 researcher. In this study the easy and simple usage of cellular phones and image processing

161 techniques will be used to develop the micro analysis of the bituminous mixture. The aim of

162 this study is to perform the micro-analysis of the bituminous mixture for different bituminous

163 mixtures with different aggregate gradations and different asphalt contents. This micro-

164 analysis is developed by using five types of cellular phones and ImageJ Software.

165 Furthermore, this study aims to investigate the image processing potential in accurate

166 measurements of the bituminous mixture volumetric design parameters (VTM, VFA, and

167 VMA). 
Materials and Methodology

170 HMA specimens with different volumetric design parameters (VTM, VMA, and VFA) were

171 prepared in the laboratory using the Superpave gyratory compactor. Different volumetric

172 values were achieved by changing the gradation of the aggregates and the asphalt content (AC

$173 \%)$ in the mixtures. As shown in Figure 1, two aggregate gradations were used; gradation

174 ARZ (above restricted zone) and gradation BRZ (below restricted zone). Nine asphalt

175 contents were used for each aggregate gradation.

176 The conventional calculation methods of computations for the volumetric design parameters

177 (VMA, VTM and VFA) of bituminous mixtures need to find some massive ratios and

178 volumetric variables of the mix. Also, the bulk specific gravity for the aggregates $\left(\mathrm{G}_{\mathrm{sb}}\right)$, the

179 bulk specific gravity of the compacted mixture $\left(\mathrm{G}_{\mathrm{mb}}\right)$ and the theoretical maximum specific

180 gravity for the loose mixture $\left(\mathrm{G}_{\mathrm{mm}}\right)$ must be determined for each HMA specimens.

181 Eighteen Superpave specimens were prepared in the laboratory using the gyratory compactor

182 (108 gyrations), with the same cylindrical shape (15 cm diameter, $11 \mathrm{~cm}$ height) but different

183 volumetric properties (VMA, VTM and VFA). According to ASTM D3203, equations1, 2 and

1843 were used to compute VMA, VTM and VFA respectively. Table 1 shows the volumetric 185 design parameters for the ARZ and the BRZ samples.

$$
\left.V M A=100\left[1-\frac{G m b\left(\frac{P s}{100}\right)}{G s b}\right)\right]
$$

Where:

$190 \quad \mathrm{VMA}=$ Voids in mineral aggregates.

$191 \mathrm{G}_{\mathrm{mb}}=$ Bulk specific gravity of compacted HMA.

$192 \mathrm{G}_{\mathrm{sb}}=$ Bulk specific gravity of aggregate.

193 Ps $=$ Aggregate, percent by total weight of $\mathrm{HMA}=100-\mathrm{AC} \%$. 


$$
V T M=100\left[1-\frac{G m b}{G m m}\right]
$$

195 Where:

$196 \quad$ VTM $=$ Voids in total mix.

$197 \mathrm{G}_{\mathrm{mb}}=$ Bulk specific gravity of compacted HMA.

$198 \mathrm{G}_{\mathrm{mm}}=$ maximum theoretical specific gravity for the loose mix.

199

200

$$
V F A=100\left[\frac{V M A-V T M}{V M A}\right]
$$

201

202

203

204

205

206

214 be flat and smooth. One slice was taken from each half. Each slice has two smooth and level

215 faces, as shown in Figure 2.

216 In this study the micro-analysis of the bituminous mixtures was developed by using Cellular

217 Phones Images (CPI) for the mixture slice faces and Image Processing Techniques (IPT),

218 specifically the ImageJ software. The image processing composed off; Superpave mixtures

219 slices, five types of cellular phones, Cellular Phone Digital Mapping Frame for Micro- 
220 Structure of Bituminous Mixture and ImageJ software as shown in Figure 3 (a). The image

221 processing procedure is shown in Figure $3(\mathrm{~b})$.

222 Five types of cellular phones were chosen to map the mixture slices faces. These types are the

223 most common using types and they have high camera resolution. Each slice face was mapped

224 by five high resolution cameras and from three heights $(25 \mathrm{~cm}, 35 \mathrm{~cm}$ and $45 \mathrm{~cm})$. Table 2

225 shows the resolution for each camera of selected cellular phones.

226 Data Acquisition system development

227 A special frame was designed and implemented to map the faces of the mixture slices. This

228 frame was designed using AutoCAD software as shown in Figure 4. The idea of this frame is

229 to standardize and facilitate the way of capturing the images of the faces of the mixture slices

230 from certain height, vertically top view and center to center (the center of the camera with the

231 center of the circular mixture slice face). As shown in Figure 4, the frame consists of top

232 plate, bottom plate, thin plate and four gradient columns. All the components were made of

233 reinforced plastic. The top plate has square shape $(25 \mathrm{~cm} \times 25 \mathrm{~cm})$ and in the middle of this

234 plate there is a circular opening for the cellular phone camera. The bottom plate also has

235 square shape $(30 \mathrm{~cm} \times 30 \mathrm{~cm})$ and in the middle there is a circular shape opening with $15 \mathrm{~cm}$

236 diameter and $3.5 \mathrm{~cm}$ in depth for the mixture slice. Under this opening there is a thin plate that

237 can be pulled, so the first slice can be replaced with second one. Between these two plates

238 there are four gradient columns with rulers on them, the scale of these rulers begins with zero

239 and ends with $45 \mathrm{~cm}$ so the height of capturing the image can be changed and fixed. The

240 upper plate can be moved by hand and leveled by using water level and it can be fixed at

241 certain height using stoppers under it, see Figure 5 (a). The cellular phone can be fixed above

242 the upper plate as the camera should be on the center's opening, see Figure 5 (b). Three

243 heights were chosen to map the mixture slices by the cellular phones $25 \mathrm{~cm}, 35 \mathrm{~cm}$, and 45

$244 \mathrm{~cm}$. 
245 In this frame, there are four gradient columns with rulers on them. The scale of these rulers

246 begins with zero and ends with $45 \mathrm{~cm}$ so the height of capturing images can be changed and

247 fixed by the stopper under the plate. Three heights were chosen to map the mixture slices by 248 the cellular phones $25 \mathrm{~cm}, 35 \mathrm{~cm}$, and $45 \mathrm{~cm}$. Each cellular phone was fixed on three different

249 heights $(25 \mathrm{~cm}, 35 \mathrm{~cm}$, and $45 \mathrm{~cm})$ using cellular phone digital mapping frame for

250 microstructure of the bituminous mixture for capturing images of the upper face and the lower

251 face of the mixture slices as shown in Figure 5. The images were analyzed by ImageJ 252 program.

253 Two slices were taken from each HMA specimen, and each slice has two faces. So, there are 254 four images for each sample. Five cameras were used to map these faces and from three 255 different heights, so for each sample there are 60 images $(2$ slices $\times 2$ faces $\times 3$ heights $\times 5$ 256 camera types). The total number of the images was 1080 images (18 sample $\times 60$ images).

257 The testing matrix was summarized in Table 3.

\section{ImageJ Software}

259 Image J is a public domain Java image processing program inspired by NIH Image for the

260 Macintosh. This software runs, either as an online applet or as a downloadable application, on 261 any computer with a Java 1.4 or later virtual machine. It can display, edit, analyze, process, 262 save and print 8-bit, 16-bit and 32-bit images. It can measure distances and angles. It can 263 create density histograms and line profile plots. It supports standard image processing 264 functions such as contrast manipulation, sharpening, smoothing, edge detection and median 265 filtering. It does scaling, rotation and flips. All analysis and processing functions are available 266 at any magnification factor. The program supports any number of windows (images)

267 simultaneously, limited only by available memory. Spatial calibration is available to provide 268 real world dimensional measurements in units such as millimeters. Density or gray scale 269 calibration is also available (Werner Bailer 2000). 
270 In this study all the images were analyzed by ImageJ program which is an open source image 271 processing program designed for scientific 2D images. This software is high usage for 272 biological tasks such as cells detection, but it was used in this study for micro-analysis of the 273 bituminous mixture and analysis for coarse aggregate shape.

274 The calibration for the Image J has been done by comparing the ImageJ results with the 275 standard software AutoCAD which is used for edge detection and find shapes areas, 276 perimeters and dimensions. Firstly, in the ImageJ software the suitable threshold value should 277 be determined using trail threshold values through analyzing the images. In 8-bit grey scale 278 images (as colored images converted into 8 bit images) there are $256\left(2^{8}\right)$ intensity 279 graduations which can be assigned to a pixel. A pixel with an intensity of 0 is black, a pixel 280 with a value of 255 is white, everything in between is a shade of grey.

281 Thresholding works by separating pixels which fall within a desired range of intensity values 282 from those which do not, (also known as 'segmentation'). Thresholding can be a very 283 effective method of measuring complex or disjointed features in an image. Image analysis 284 software has no intuitive moves up its sleeve; it will take all the information in an image and 285 treat it literally. All the threshold values were tested for determining the suitable value which 286 gives the highest accuracy percentage for the particles areas by comparing it with AutoCAD 287 areas. In most of the images, if the threshold values were between 0 and 100, then the white 288 color will be prominent and small particles will not be spotted. On the other hand, if the 289 values were between 150 and 255, then the black color will prevail. So the suitable threshold 290 value should be in the range of 100-150. The checked threshold values were $100,105,110$, $291115,120,125,130,135,140,145,150$ and the average value 127.

292 ImageJ software was calibrated by computing the accuracy percentage and the error 293 percentage of measurements of areas and perimeters for three kinds of shapes. The AutoCAD 294 software was used to compute the actual areas and perimeters for these shapes. These kinds of 
295 shapes are: regular shapes, irregular shapes, and selected 45 aggregate irregular shapes from a

296 selected HMA slice, see Figure 6. The selected regular shapes were: circle, triangle, trapezoid

297 and rectangle, see Figure 6 (a). The irregular shapes, in Figure 6 (b), were not from mixture

298 cutting face, they were random irregular shapes similar to the aggregates shapes the mixture

299 slice face. Fifteen random irregular shapes were classified into three groups based on their

300 areas; 5 large particles, 5 medium particles and 5 small particles. For the regular and irregular

301 shapes, the Image J accuracy percentage for the measured areas was almost $100 \%$ and the

302 error percentage was almost zero. Figure 6 (c) shows the selected forty-five irregular

303 aggregate particles. A comparison between the collected data from AutoCAD with the data

304 from ImageJ analysis of the areas and perimeters for the regular shapes was shown Figure 7

305 (a) and Figure 7 (b), respectively. Figure7 (c) and Figure 7 (d) show the comparison between

306 collected data from AutoCAD with data from ImageJ analysis of the areas and perimeters for

307 the irregular shapes.

308 Forty-Five of irregular shapes of aggregate were selected from mixture slice image. The

309 image in Figure 8 was captured by cellular phone camera with high resolution. The selection

310 of 45 particles was for calibration of ImageJ software and selecting the suitable thresholding

311 value. In Typical HMA slice's image, the maximum number of detectable large aggregate

312 particles was 15 , similar particle numbers of medium and small particle sizes were selected

313 with a total number of 45 particles. It shows the whole particles in the mixture clearly. These

314 particles have irregular shapes with known areas and perimeters by using AutoCAD software.

315 The irregular shapes were classified upon their areas into three classes. The large particles

316 have area more the $80 \mathrm{~mm}^{2}$, the medium particles have areas between $20 \mathrm{~mm}^{2}$ and $80 \mathrm{~mm}^{2}$

317 and the small particles have areas less than $20 \mathrm{~mm}^{2}$. Figure 8 shows the selected fifteen large

318 particles (Figure 8(a)), fifteen medium particles (Figure 8(b)) and fifteen small particles

319 (Figure 8(c)) according to their areas and perimeters. 
320 The threshold value has been changed from 100 to 150 in an increment of 5, and including the

321 average value 127. This changing of the threshold value for detection the irregular areas of the

322 aggregates in mixture slice face was effecting on the accuracy percentage, and the error 323 percentage. The ImageJ analysis was done for this mixture image at each threshold value.

324 Figure 9 shows how to analyze the mixture particles after selection the threshold value.

325 Mixture slices face before and after Thresholding is shown in Figure 9(a) while the area 326 detection by Image J is shown in Figure 9(b).

327 This procedure was repeated for 12 times changing the threshold value. The results showed 328 that the suitable threshold value for analyzing the images is 130 . This value scored the highest 329 accuracy percentage and the lowest error percentage for the measurements of areas and 330 perimeters of the aggregate particles by ImageJ software. Figures 10 shows an example of 331 accuracy (Figure 10(a)) and error percentages (Figure 10(b)) for the large particles.

\section{ImageJ Analysis Variables}

334

The 1080 cellular phone images, from the five types of cellular phones as mentioned before and on three heights of capturing, were analyzed by ImageJ software using threshold value

337 130. The ImageJ analysis results for the one mixture slice face were got out in table and a

338 summary for the columns data will be shown at the end of each column. This summary 339 includes mean, standard deviation (SD), minimum, and maximum values for the data in one

340 column. From this summary, the mean of the particle areas and the mean of the particle 341 perimeters were selected variables for the models.

342 Also, another summary will be got out from the analysis. It shows the count of the particles

343 that were detected by ImageJ in the mixture slice face and the total area of these particles.

344 These two variables also selected for the models. Also, the percentage of the total areas of 345 detected particles from the whole circular area of the mixture slice was selected to be in the 
346 models. These data collected from the five cellular phone types and on the three heights. The

347 selected variables were the average values, from the four images for each mixture (i.e. each

348 image at different depth of the HMA sample, with slightly different results for each variable).

\section{$350 \quad$ Analysis and Results}

351 The main objective of this study is to make image processing outcomes more reliable rather

352 than being always dependent on laboratory test results only. New models for the volumetric

353 design parameters were integrated from the results of ImageJ analysis for the mixtures

354 images. SPSS software was used to analyze the ImageJ data for all cellular phones images

355 from all types of cameras and from three heights $(25 \mathrm{~cm}, 35 \mathrm{~cm}$ and $45 \mathrm{~cm})$ asphalt concrete

356 mixtures slices. Two specimens were ignored which have $\mathrm{AC} \%=7 \%$ because there was

357 significant difference in the values of needed variables (count of particles, total area of 358 particles, area percentage and average perimeters of particles) in the four images of same

359 Superpave specimen and from the same height due to the bleeding that happened on the

360 specimen with the time before cutting it into slices. So the data were collected from 16

361 Superpave specimens. The results show that the cellular phone type D gave the most accurate

362 volumetric data about the mixtures even though it does not have the highest resolution.

363 However, these images (using type D cellular phone) show more contrast between the white

364 and black colors in ImageJ software, as shown in Figure 9 (a). The colored image should be

365 converted to 8-bit image to be analyzed by ImageJ software. The data from ImageJ analysis

366 for 16 mixtures slices imaged from cellular phone type D were analyzed statistically by SPSS

367 software using Backward method and Multiple Non-linear regression to integrate the

368 volumetric design parameters models (VTM, VMA and VFA).

369 The integrated models were followed the formula below:

$$
Y=f(x 1, x 2, x 3, x 4, x 5)
$$


370 Where:

$371 \mathrm{Y}=\mathrm{VMA}, \mathrm{VTM}$ or VFA.

372 VMA=Void in Mineral Aggregates. (\%)

$373 \quad$ VTM $=$ Void in Total Mix (\%)

$374 \quad$ VFA $=$ Voids Filled with Asphalt (\%)

$375 \times 1=\log$ count $=\log$ count of detectable aggregate particles that have been detected by ImageJ

376 (Number).

$377 \times 2=\log$ total area $=\log$ total area of the selected aggregate particles $\left(\mathrm{mm}^{2}\right)$.

$378 \times 3=\log$ area percentage $=\log$ area percentage of total area of aggregate particles to the total

379 area of the cutting face $(\%)$.

$380 \times 4=$ mean area $=$ mean area of the aggregate particles $\left(\mathrm{mm}^{2}\right)$.

$381 \times 5=$ mean perimeter $=$ mean perimeter of the aggregate particles $(\mathrm{mm})$.

382 The integrated models in this research were:

383 VMA $\%=198.428+19.482 \log$ count $-134.121 \log \%$ area +0.343 mean area

384 VTM\% $=294.656+108.991 \log$ count $-55.194 \log$ total area $-226.702 \log$ area $\%+$

3851.555 mean area

386

387

VFA $\%=-207.367-759.061 \log$ count $+654.656 \log$ total area -10.318 mean area

388

389 Equation 4 is the first integrated model for computation of VMA of the HMA mixtures. The 390 coefficient of determination $\mathrm{R}^{2}$ was 0.895 , the adjusted $\mathrm{R}^{2}$ was 0.868 , and the standard error 391 of the estimate (SE) was 0.47 . The count of the detectable aggregate particles represents the

392 volume of the aggregate in the mixture which affects the VMA value, if the count increase the

393 VMA value will increase. On the other hand, the area percentage of total area of aggregate 394 particles to the total area of the cut face affect negatively on the VMA value, which is 395 logically true. 
396 Equation 5 is for computation of VTM of the HMA mixtures and for this model $\mathrm{R}^{2}$ was 0.895 ,

397 the adjusted $\mathrm{R}^{2}$ was 0.857 , and $\mathrm{SE}$ was $1.283 \%$. The count of the aggregate particles

398 represents the volume of the aggregate in the mixture which affect the VTM value, if the

399 count increase the VTM value will increase. On the other hand, the total area of the aggregate

400 particles and the area percentage of total area of aggregate particles to the total area of the cut

401 face affect negatively on the VTM. Therefore, when the particles in the mixture have different

402 sizes (large, medium and small) this will make the total area of the cut particles increase as

403 well as their percentage area to the total cut face, and this will decrease the total voids in the

404 mixture (VTM). In the equation 4 and 5, the most effective variable was the percentage of the

405 particles' areas to the total cutting face area.

406 Equation 6 is the integrated model for computation of FVA of the HMA mixtures. With $\mathrm{R}^{2}$

407 was 0.896 , the adjusted $\mathrm{R}^{2}$ was 0.845 , and SE $7.689 \%$. Here, the model shows that the count

408 of the particles will effect on the VFA negatively, if the count increase the VFA will decrease.

409 But, the total area and the mean area effect positively on the VFA. This means that the voids

410 filled with asphalt will increase if the aggregate size increase. The effective variable in VFA

411 model is the count of the detectable aggregate particles. Table 4 and Figure 11 show that

412 there are slight differences between the predicted values (using the new models) and the

413 actual values (using conventional methods) of VMA, VTM and VFA. Table 5 shows the

414 minimum, maximum and the average differences between the actual and the predicted values

415 of VMA, VTM and VFA and the error percentages.

416 The linearity assumption checks for the models are shown in Figures 12. It is clear that all the

417 residuals are clustered around the line suggesting that the assumption of normality has been

418 met. On other hand the predicted values are normally scattered around the center line which is

419 another prove of the normality assumption.

420 


\section{Conclusions}

422 The usage of image processing is growing fast in many aspects of science and engineering 423 applications. The aim of this study is to use cellular phone cameras in one of the civil 424 engineering applications. The micro-analysis of bituminous mixtures, namely is to determine volumetric design parameters of bituminous mixtures and identify some shape properties of coarse aggregate. This study reduces and eliminates the error associated with the assumptions traditionally made in the micro structure and improves the validity of integrated models that can fundamentally describe the behavior of HMA. Furthermore, the incorporation of the microstructure into computational simulation demonstrates its flexibility and power.The outcomes of this study were promising and there is a great potential for development and improvements for the micro-analysis of the bituminous mixtures. Therefore, this technique will not require the expensive $\mathrm{x}$ - ray computed tomography equipment. The study provided the simple and easy usage of cellular phones and ImageJ software. This system has a potential to bridge the gap between conventional or manual procedures and fully automated calculation methods. From the statistical analysis and comparisons made in this study, the following conclusions could be drawn:

1- It was found that the usage of the image analysis technique for VMA, VTM, and VFA of bituminous mixture computation is practical. The experiment emphasized the importance of the application of cellular phones and image-processing operations to enhance aggregate edge detection, count the number of the particles, and compute their areas and perimeters. The result did not depend on the resolution of the cellular phone camera, it depends on the camera contrast after converting the colored image to 8-bit image (black and white image). This conversion is to be able to use the ImageJ software to analyze the images. 
2- New models for the volumetric design parameters were integrated using the Image J outcomes variables and backward linear regression method.

3- ImageJ software provides a powerful tool to accurately and nondestructively quantify the understanding of its behavior as well as the interpretation of experimental results.

The usage of cellular phones and Image processing techniques, a strongly emerging technology, have proven to be capable of objectively quantifying volumetric design parameters of the bituminous mixture and coarse aggregates shape characteristics.

\section{Recommendations}

456 The results of this research led to the conclusion that there is a highly potential of using 457 cellular phone image and image processing technique for micro-analysis of bituminous 458 mixture. The potential can most fully be exploited if further research is directed to the 459 following recommendations:

460 1- Further research is recommended to use cellular phone images and image processing for 461 micro-analysis of the bituminous mixtures that uses dark aggregates in the HMA such $462 \quad$ as basalt.

463 2- Further research is recommended to build a cellular phone application which calculates 464 the volumetric design parameters automatically on the phone without using the $465 \quad$ computer software.

466 3- Vertical cross sections of the bituminous mixtures were not involved in this research. A 467 further investigation could be done to highlight the significance of the vertical cross 
468

469

470

471

472

473

474

475

476

477

478

479

480

481

482

483

484

section in the micro-analysis of the mixture and if they are affecting the values of volumetric design parameters.

4- Further research is recommended for determining the degree of compaction of the mixture based on the cellular phone images and image processing for determination the changes in the center to center distances between the aggregate particles before and after the compaction.

5- Further research is recommended for determining the aggregates gradation used in the mixtures by using cellular phone images and image processing to determine the percentage of the passing through or retained aggregates on each sieve according to the sieve size.

\section{Acknowledgement}

This article is part of Master Degree Thesis at Jordan University of Science and Technology (JUST). A full report of the research work can be found at Alawneh (2016) at the College of Graduate Studies at JUST under the title: "The Micro-Analysis of Bituminous Mixture Using Cellular Phones and Image Processing Techniques" and this research was supported by the deanship of scientific research at JUST (Research No. 154/2015). 


\section{References}

Alawneh, M. M. 2016. Micro-analysis of bituminous mixture using cellular phones and image processing techniques, MSc thesis, Jordan University of Science \& Technology, Irbid, Jordan.

Asphalt Institute. 1988. Mix design method for asphalt concrete and other hot mix types. Manual Series Number 2 (MS-2), Asphalt Institute, Lexington, KY.

ASTM, Standard Test Method for Percent Air Voids in Compacted Dense and Open Bituminous Paving Mixtures, D3203-94. Annual Book of ASTM Standards, Vol. 04.03, (American Society for Testing and Materials, West Conshohocken, PA).

Bessa, L., Castelo Branco, V., and Soares, J. 2012. Evaluation of different digital image processing software for aggregates and hot mix asphalt characterizations. Construction and Building Materials, 37, 370-378.

Obaidat M.T., Al-Masaeid H.R., Gharaybeh, F. and Khedaywi T.S. 1998. An innovative digital image analysis approach to quantify the percentage of voids in mineral aggregates of bituminous mixtures. NRC Canada, J. Civ. Eng. 25, 1041-1049.

Tashman L., Wang L., and Thyagarajan S. 2011. Microstructure characterization for modeling HMA behavior using imaging technology. Road Materials and Pavement Design, 8:2, 207-238,

Tashman L., Masad E., Angelo J. D., Bukowski J., and Harman T. 2002. X-ray tomography to characterize air void distribution in Superpave gyratory compacted specimens. International Journal of Pavement Engineering, 3(1), 1928. 
Tashman L., Masad E., Peterson B., and Saleh H. 2001. Internal structure analysis of asphalt mixes to improve the simulation of Superpave gyratory compaction to field conditions. Journal of the Association of Asphalt Paving Technologists, $70,605-645$.

You, Z. and Buttlar, W. G. 2004. Discrete element modeling to predict the modulus of asphalt concrete mixtures. Journal of Materials in Civil Engineering, 16(2), 140146.

Wang, L.B., Frost, J.D. and Shashidhar, N. 2001. Microstructure study of West track mixes from X-Ray tomography images. Transportation Research Record 1767, Transportation Research Board, National Research Council, Washington, D.C., $85-94$.

Werner Bailer. 2000. Writing ImageJ PlugIns - A Tutorial. Version 1.0 - Oct. 18.

Vadood, M., Johari, M. and Rahaei, A. 2014. Introducing a simple method to determine aggregate gradation of hot mix asphalt using image processing. International Journal of Pavement Engineering, 15(2), 142-150.

Zelelew, H.M., and Papagiannakis A.T. 2007. A Volumetrics thresholding algorithm for processing asphalt concrete X-ray CT images. International Journal of Pavement Engineering.

ZhanpingYou, Sanjeev Adhikari, and Karl Peterson. 2013. Multi- Phase Characterization of Asphalt Concrete using X- ray Micro fluorescence. International Journal of Pavement Research and Technology, 6(2), 117-122. 


\section{$\underline{\text { List of Tables }}$}

Table 1: Volumetric Properties for the HMA specimens.

Table 2: The Selected Cellular phones.

Table 3: Testing Matrix.

Table 4: Differences between the actual values and the predicted values of the mixtures' volumetric parameters (VMA, VTM and VFA) and the error percentages.

Table 5: Minimum, maximum and average values of the differences and error percentages between the actual and predicted values of VMA, VTM and VFA for 16 mixtures.

\section{$\underline{\text { List of Figures }}$}

Figure 1: The 0.45 power gradations curve of $12.5 \mathrm{~mm}$ (ARZ and BRZ Gradations).

Figure 2: Cutting the sample into two slices.

Figure 3: The image processing components and procedure.

Figure 4: AutoCAD design For the Frame.

Figure 5: The Cellular phone digital mapping frame for the microstructure of the bituminous mixture.

Figure 6: Three kinds of shapes for ImageJ calibration.

Figure 7: Comparison between the collected data from AutoCAD with data from ImageJ analysis for the regular and irregular shapes.

Figure 8: The sizes' classification of the selected forty-five aggregate particles.

Figure 9: Thresholding and particle analysis.

Figure 10: An example for the percentage of accuracy and error for the computation of large particles areas by the ImageJ at the threshold values from 100 to 150 .

Figure 11: Comparison between the predicted value of VMA, VTM and VFA using the new models, respectively, and the actual value from the conventional methods. 
Figure 12: The linearity assumption checks for proposed models. 
Table 1: Volumetric Properties for the HMA specimens.

\begin{tabular}{|c|c|c|c|c|c|c|c|c|}
\hline $\begin{array}{c}\text { Aggregate } \\
\text { Gradation }\end{array}$ & $\begin{array}{c}\text { Sample } \\
\text { No. }\end{array}$ & $\mathbf{A C} \%$ & $\mathbf{P}_{\mathbf{s}} \%$ & $\mathbf{G}_{\mathbf{m m}}$ & $\mathbf{G}_{\mathbf{m b}}$ & $\mathbf{V T M} \%$ & VFA\% & VMA\% \\
\hline \hline \multirow{5}{*}{ ARZ } & 1 & 4 & 96 & 2.463 & 2.284 & 7.27 & 48.14 & 14.01 \\
\cline { 2 - 9 } & 2 & 4.5 & 95.5 & 2.452 & 2.303 & 6.08 & 55.81 & 13.75 \\
\cline { 2 - 9 } & 3 & 4.75 & 95.25 & 2.443 & 2.311 & 5.4 & 60.518 & 13.677 \\
\cline { 2 - 9 } & 4 & 5 & 95 & 2.427 & 2.334 & 3.83 & 70.63 & 13.05 \\
\cline { 2 - 9 } & 5 & 5.25 & 94.75 & 2.417 & 2.337 & 3.31 & 74.856 & 13.164 \\
\cline { 2 - 9 } & 6 & 5.5 & 94.5 & 2.408 & 2.339 & 2.87 & 78.49 & 13.32 \\
\cline { 2 - 9 } & 7 & 6 & 94 & 2.398 & 2.348 & 2.09 & 84.49 & 13.45 \\
\cline { 2 - 9 } & 8 & 6.5 & 93.5 & 2.376 & 2.349 & 1.14 & 91.81 & 13.87 \\
\hline \multirow{5}{*}{ BRZ } & 9 & 7 & 93 & 2.364 & 2.346 & 0.76 & 94.73 & 14.44 \\
\cline { 2 - 9 } & 10 & 4 & 96 & 2.514 & 2.193 & 12.77 & 24.24 & 16.85 \\
\cline { 2 - 9 } & 11 & 4.5 & 95.5 & 2.48 & 2.213 & 10.77 & 34.88 & 16.53 \\
\cline { 2 - 9 } & 12 & 4.75 & 95.25 & 2.473 & 2.224 & 10.07 & 38.357 & 16.336 \\
\cline { 2 - 9 } & 13 & 5 & 95 & 2.465 & 2.244 & 8.97 & 43.28 & 15.81 \\
\cline { 2 - 9 } & 15 & 5.5 & 94.5 & 2.455 & 2.278 & 7.21 & 51.87 & 14.98 \\
\cline { 2 - 8 } & 16 & 6 & 94 & 2.429 & 2.285 & 5.93 & 60.92 & 15.17 \\
\cline { 2 - 8 } & 17 & 6.5 & 93.5 & 2.402 & 2.334 & 2.83 & 79.50 & 13.81 \\
\cline { 2 - 8 } & 18 & 7 & 93 & 2.378 & 2.333 & 1.89 & 86.78 & 14.31 \\
\hline
\end{tabular}


Table 2: The Selected Cellular phones.

\begin{tabular}{|c|c|c|c|c|}
\hline $\begin{array}{c}\text { Cellular } \\
\text { phone } \\
\text { type }\end{array}$ & $\begin{array}{c}\text { Image Width } \\
\text { (pixels) }\end{array}$ & $\begin{array}{c}\text { Image Height } \\
\text { (pixels) }\end{array}$ & Resolution & $\begin{array}{c}\text { Resolution in } \\
\text { Mega Pixels }\end{array}$ \\
\hline Type A & 3840 & 2160 & $3840 \times 2160$ & 8.29 \\
\hline Type B & 3264 & 2448 & $3264 \times 2448$ & 7.99 \\
\hline Type C & 3552 & 2000 & $3552 \times 2000$ & 7.10 \\
\hline Type D & 2448 & 2448 & $2448 \times 2448$ & 5.99 \\
\hline Type E & 2688 & 1520 & $2688 \times 1520$ & 4.08 \\
\hline
\end{tabular}


Table 3: Testing Matrix.

\begin{tabular}{|c|c|c|c|}
\hline Camera Type & 5 & $\begin{array}{c}\text { Type A, Type B, Type C, type D and } \\
\text { Type E }\end{array}$ & \multirow{6}{*}{$\begin{array}{c}5 \times 1 \times 18 \times 2 \times 2 \times 3=1080 \\
\text { images }\end{array}$} \\
\hline Resolutions & 1 & One High resolution for each camera & \\
\hline $\begin{array}{l}\text { Superpave } \\
\text { samples }\end{array}$ & 18 & (9) $\mathrm{AC} \% \mathrm{x}(2)$ Gradation & \\
\hline Slices & 2 & (2) slices from each sample & \\
\hline Faces & 2 & (2) smooth and flat faces for each slice & \\
\hline $\begin{array}{c}\text { Capturing } \\
\text { height }\end{array}$ & 3 & $25 \mathrm{~cm}, 35 \mathrm{~cm}$ and $45 \mathrm{~cm}$ & \\
\hline
\end{tabular}


Table 4: Differences between the actual values of the mixtures' volumetric parameters (VMA, VTM and VFA) and the predicted ones.

\begin{tabular}{|c|c|c|c|c|c|c|c|c|c|c|c|c|}
\hline $\begin{array}{c}\text { Sample } \\
\text { Number }\end{array}$ & $\begin{array}{c}\text { VMA } \\
\text { Actual } \\
\end{array}$ & $\begin{array}{c}\text { VMA } \\
\text { Predicted }\end{array}$ & Difference & $\begin{array}{c}\text { Error } \\
\%\end{array}$ & $\begin{array}{c}\text { VTM } \\
\text { Actual } \\
\end{array}$ & $\begin{array}{c}\text { VTM } \\
\text { Predicted } \\
\end{array}$ & Difference & $\begin{array}{c}\text { Error } \\
\%\end{array}$ & $\begin{array}{c}\text { VFA } \\
\text { Actual } \\
\end{array}$ & $\begin{array}{c}\text { VFA } \\
\text { Predicted } \\
\end{array}$ & Difference & $\begin{array}{c}\text { Error } \\
\% \\
\end{array}$ \\
\hline 1 & 14.01 & 14.14 & 0.12 & 0.87 & 6.53 & 6.72 & 0.19 & 3.44 & 55.14 & 50.30 & 4.84 & 8.78 \\
\hline 2 & 13.75 & 13.74 & 0.01 & 0.07 & 5.52 & 5.73 & 0.21 & 3.59 & 59.81 & 58.59 & 1.21 & 2.03 \\
\hline 3 & 13.68 & 13.55 & 0.14 & 1.00 & 5.85 & 5.39 & 0.46 & 16.06 & 58.52 & 62.18 & 3.66 & 6.26 \\
\hline 4 & 13.05 & 13.29 & 0.24 & 1.85 & 2.87 & 2.84 & 0.02 & 0.42 & 76.63 & 77.18 & 0.55 & 0.71 \\
\hline 5 & 13.16 & 13.55 & 0.38 & 2.90 & 5.69 & 5.39 & 0.29 & 9.76 & 60.86 & 62.18 & 1.33 & 2.18 \\
\hline 6 & 13.32 & 13.72 & 0.40 & 2.97 & 3.00 & 2.69 & 0.31 & 25.37 & 78.49 & 76.38 & 2.11 & 2.68 \\
\hline 7 & 13.45 & 12.88 & 0.57 & 4.24 & 1.23 & 1.04 & 0.19 & 16.46 & 85.49 & 91.67 & 6.18 & 7.23 \\
\hline 8 & 13.87 & 13.36 & 0.51 & 3.67 & 1.14 & 1.70 & 0.57 & 4.84 & 91.81 & 84.62 & 7.19 & 7.83 \\
\hline 9 & 16.85 & 16.66 & 0.20 & 1.17 & 11.72 & 11.65 & 0.07 & 0.61 & 30.24 & 30.39 & 0.15 & 0.50 \\
\hline 10 & 16.53 & 16.66 & 0.13 & 0.77 & 11.77 & 11.86 & 0.10 & 0.97 & 30.88 & 28.48 & 2.40 & 7.76 \\
\hline 11 & 16.34 & 15.91 & 0.42 & 2.58 & 10.07 & 9.51 & 0.56 & 7.02 & 39.36 & 40.25 & 0.89 & 2.27 \\
\hline 12 & 15.81 & 15.19 & 0.61 & 3.88 & 7.96 & 7.21 & 0.76 & 8.89 & 52.28 & 51.80 & 0.48 & 0.91 \\
\hline 13 & 15.62 & 15.30 & 0.32 & 2.05 & 8.51 & 7.38 & 1.13 & 13.81 & 50.91 & 52.76 & 1.85 & 3.63 \\
\hline 14 & 14.98 & 15.58 & 0.60 & 4.01 & 8.21 & 8.07 & 0.14 & 2.03 & 48.87 & 46.27 & 2.60 & 5.33 \\
\hline 15 & 15.17 & 15.24 & 0.07 & 0.44 & 6.99 & 6.87 & 0.13 & 3.18 & 54.92 & 55.12 & 0.20 & 0.36 \\
\hline 16 & 13.81 & 14.59 & 0.78 & 5.65 & 3.97 & 4.46 & 0.49 & 12.41 & 73.50 & 76.22 & 2.72 & 3.70 \\
\hline
\end{tabular}


Table 5: Minimum, maximum and average values of the differences and error percentages between the actual and predicted values of VMA, VTM and VFA for 16 mixtures.

\begin{tabular}{|l|c|c|c|c|c|c|}
\hline Difference & VMA & Error\% & VTM & Error\% & VFA & Error\% \\
\hline Minimum & 0.01 & 0.07 & 0.02 & 0.42 & 7.19 & 7.83 \\
\hline Maximum & 0.78 & 5.65 & 1.13 & 13.81 & 0.15 & 0.50 \\
\hline Average & 0.34 & & 0.35 & & 2.40 & \\
\hline
\end{tabular}




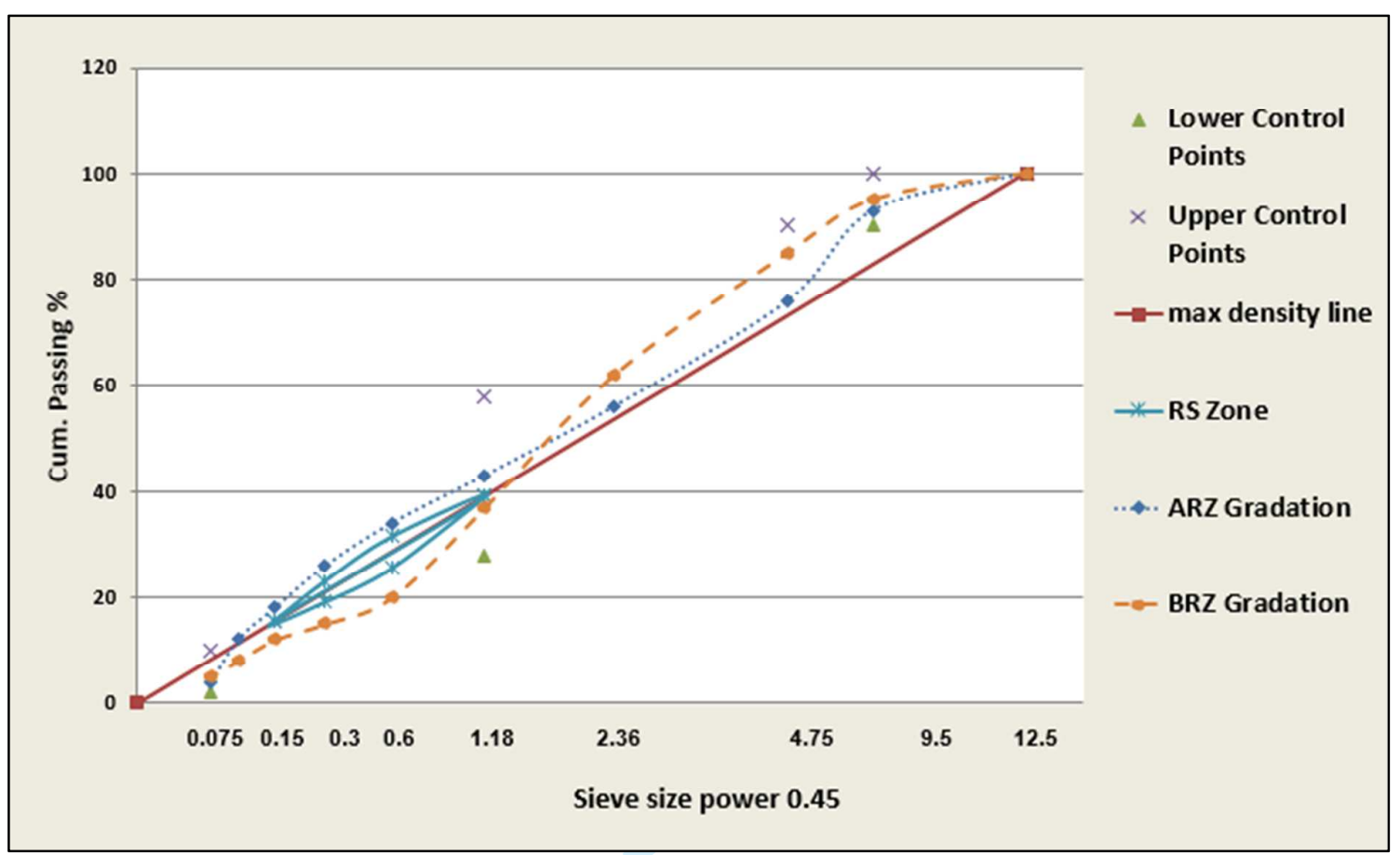

Figure 1: The 0.45 power gradations curve of $12.5 \mathrm{~mm}$ (ARZ and BRZ Gradations). 


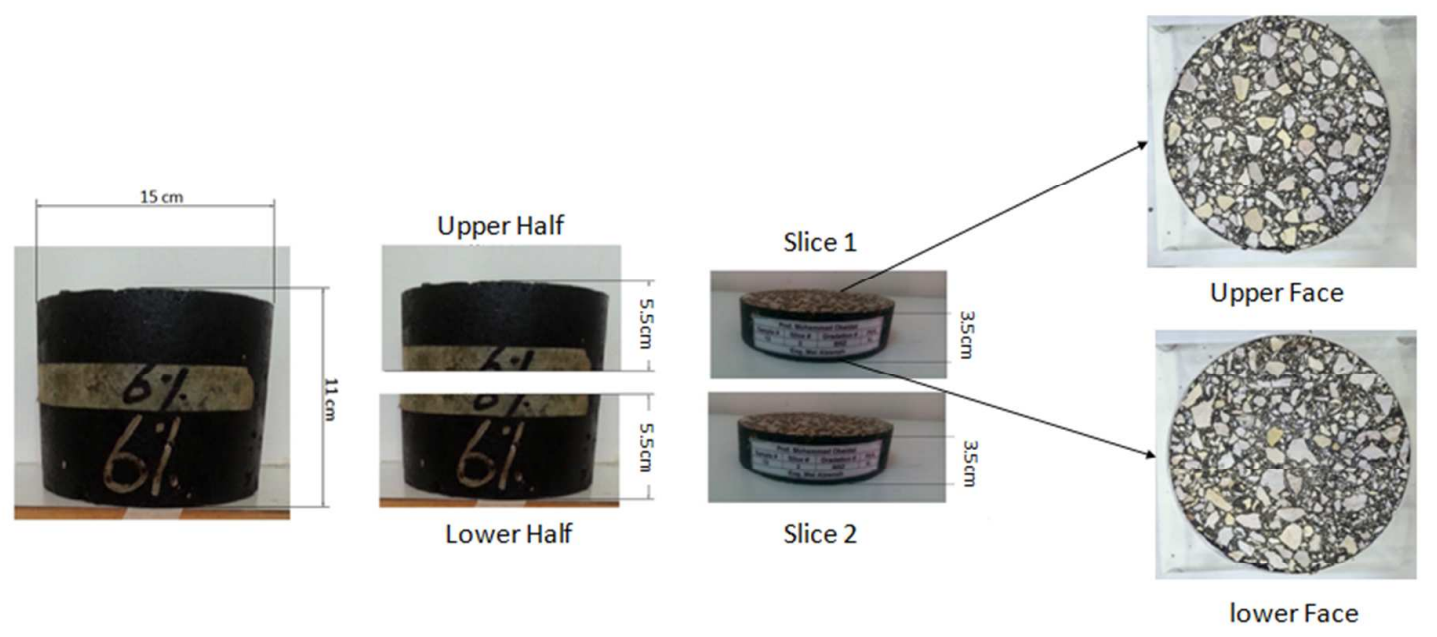

Figure 2: Cutting the sample into two slices. 


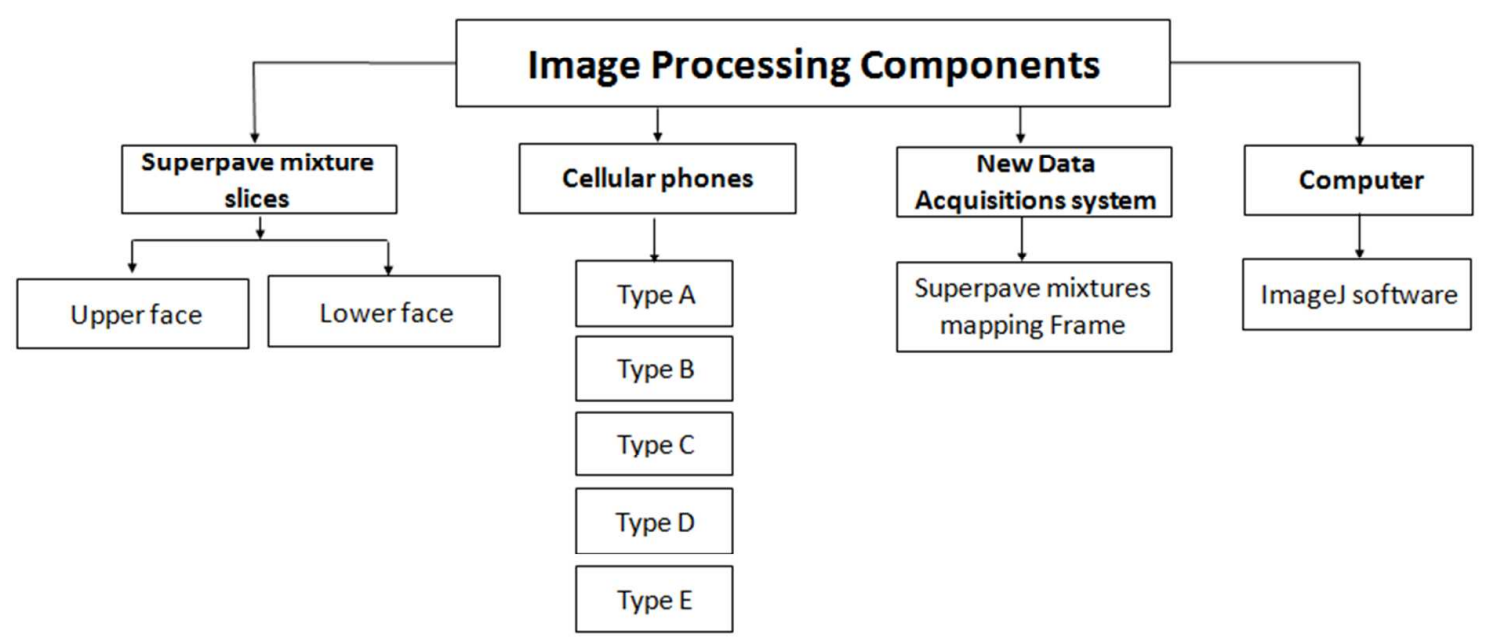

(a) The image processing components.

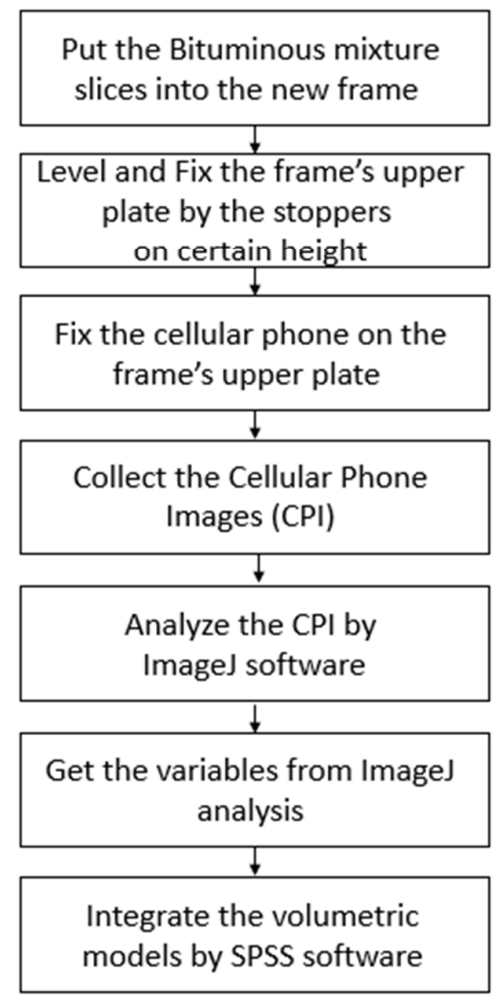

(b) The image processing procedures.

Figure 3: The image processing components and procedures. 

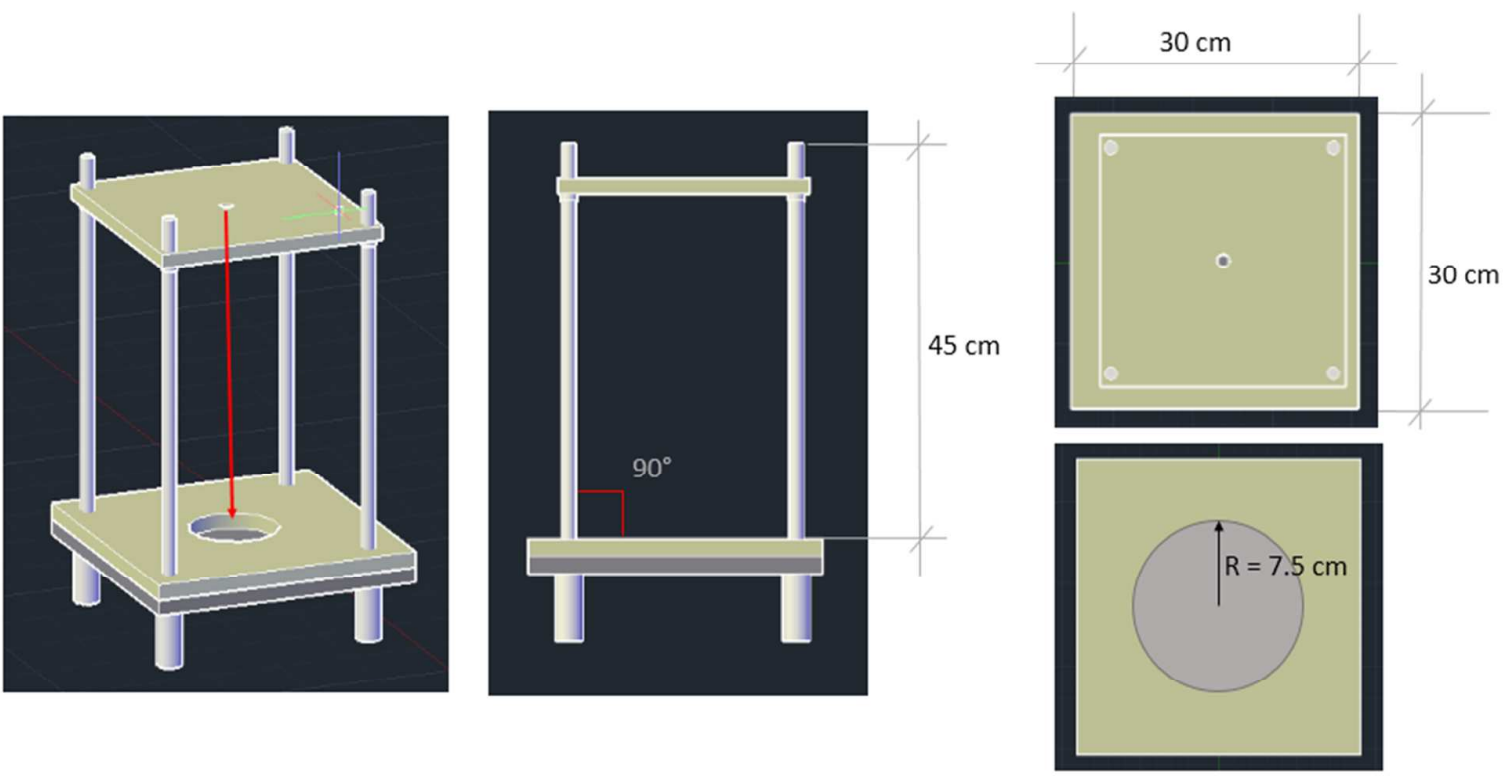

Figure 4: AutoCAD design For the Frame. 


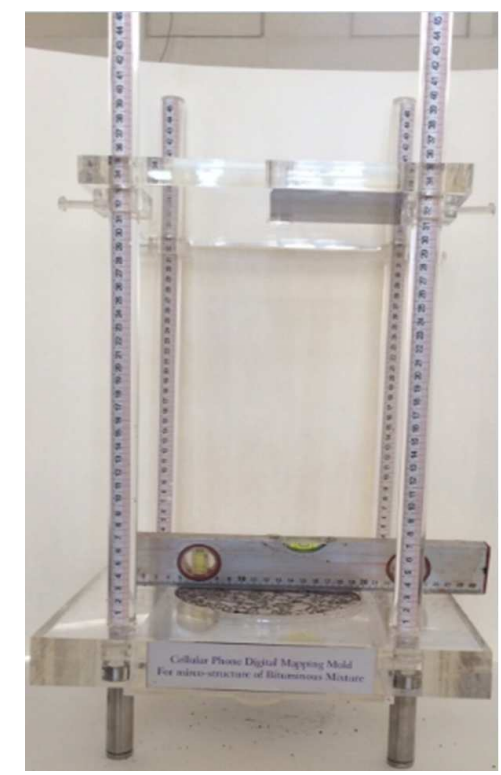

(a) Cellular Phone Digital Mapping Frame for Micro-Structure of Bituminous Mixture.

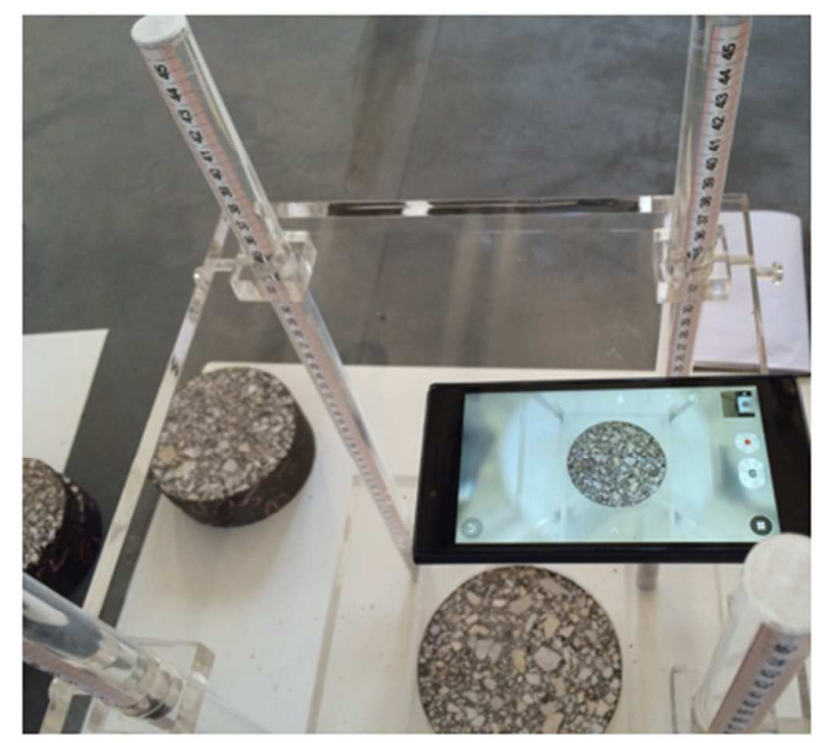

(b)Fixing the cellular phone on the upper plate at certain height.

Figure 5: The Cellular phone digital mapping frame for the microstructure of the bituminous mixture. 


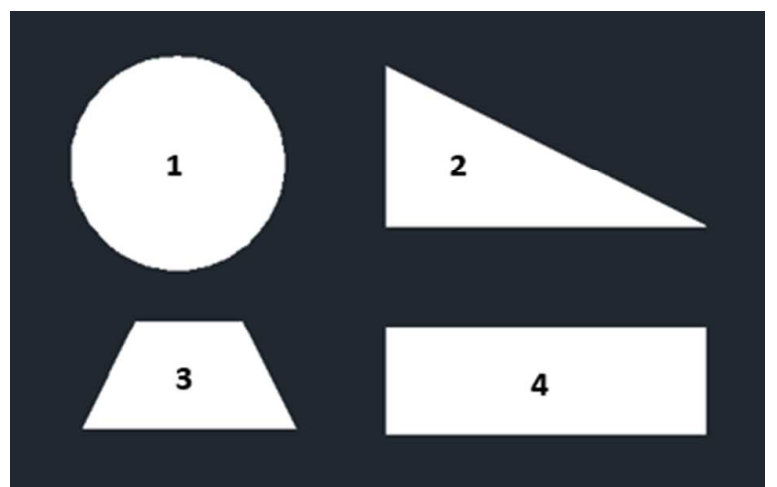

(a) Four Regular shapes.

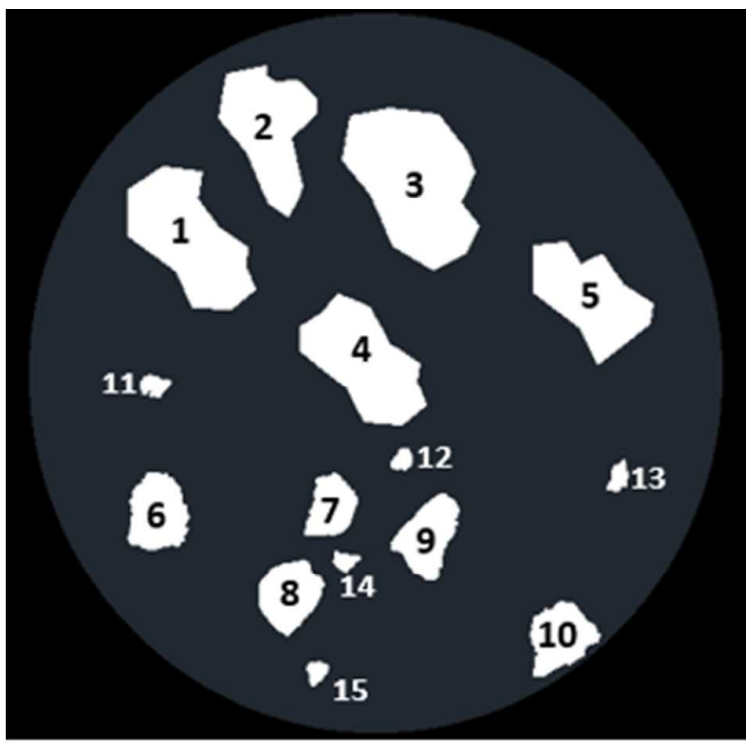

(b) Fifteen irregular shapes.

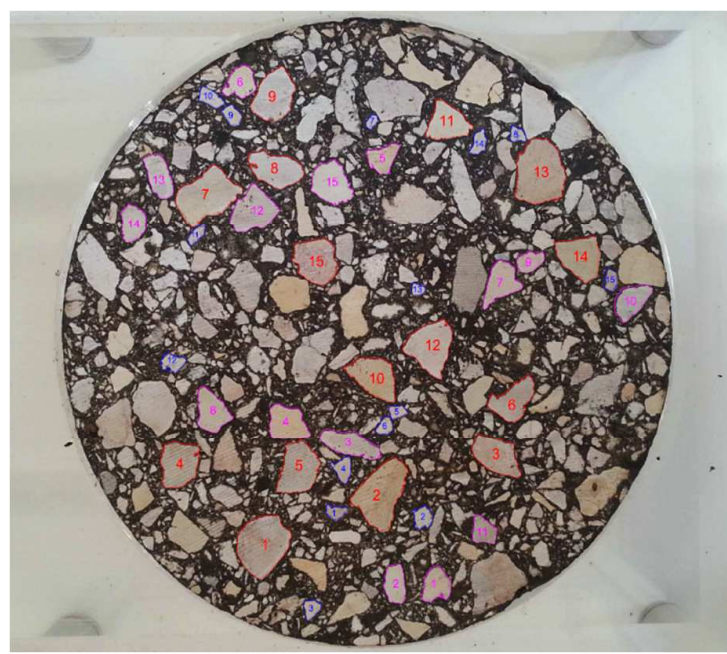

(c) The Selected forty-five irregular aggregate particles.

Figure 6: Three kinds of shapes for ImageJ calibration. 


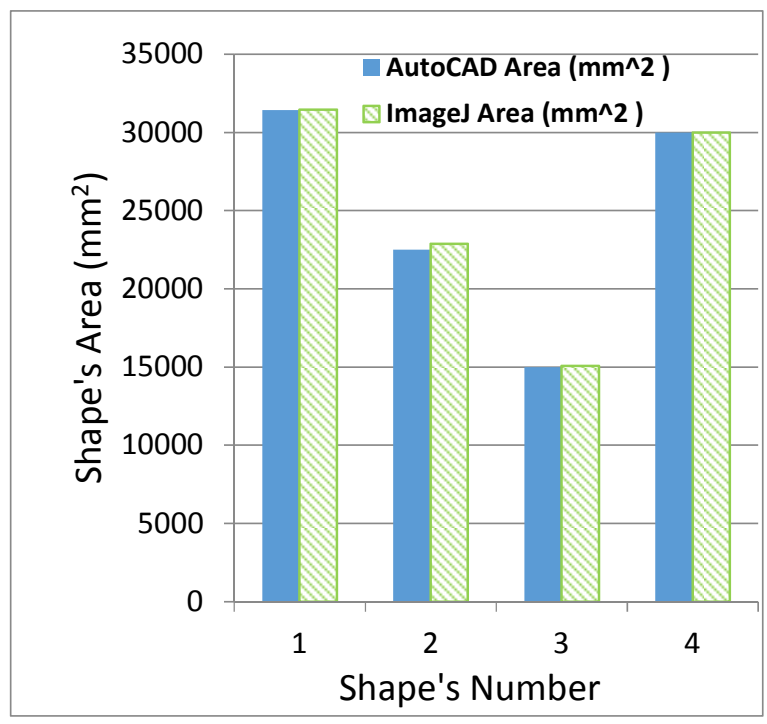

(a) Comparison between regular shapes areas from AutoCAD and ImageJ.

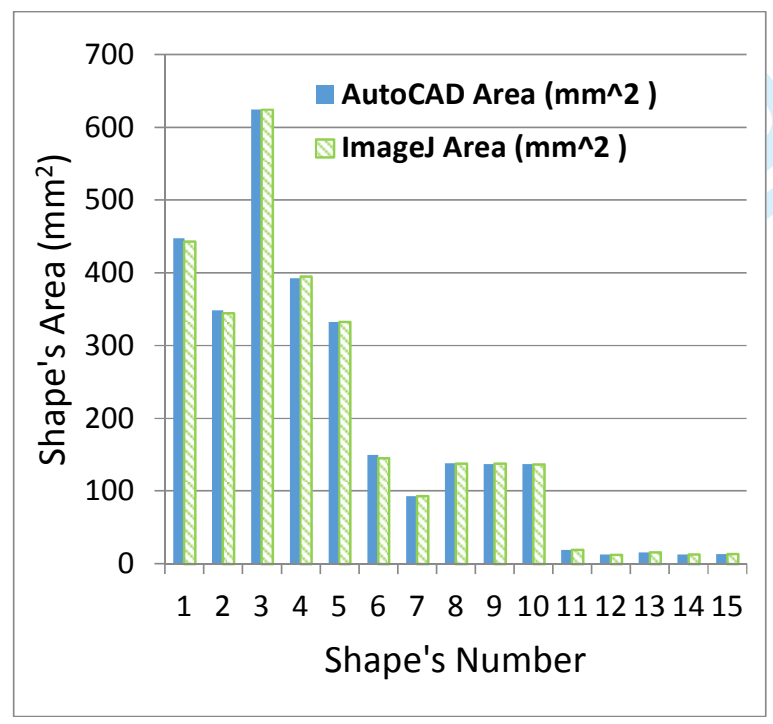

(c) Comparison between irregular shapes areas from AutoCAD and ImageJ.

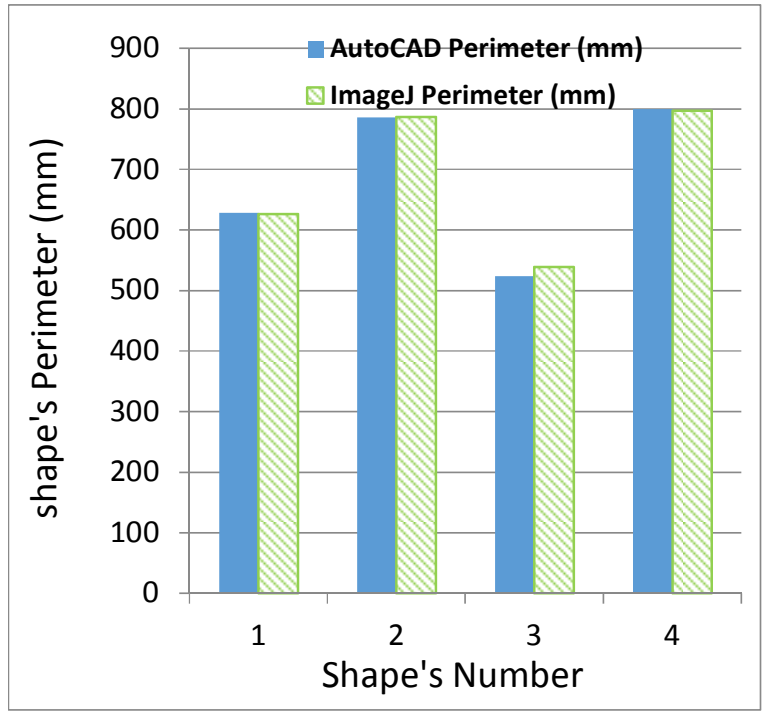

(b) Comparison between regular shapes perimeters from AutoCAD and ImageJ.

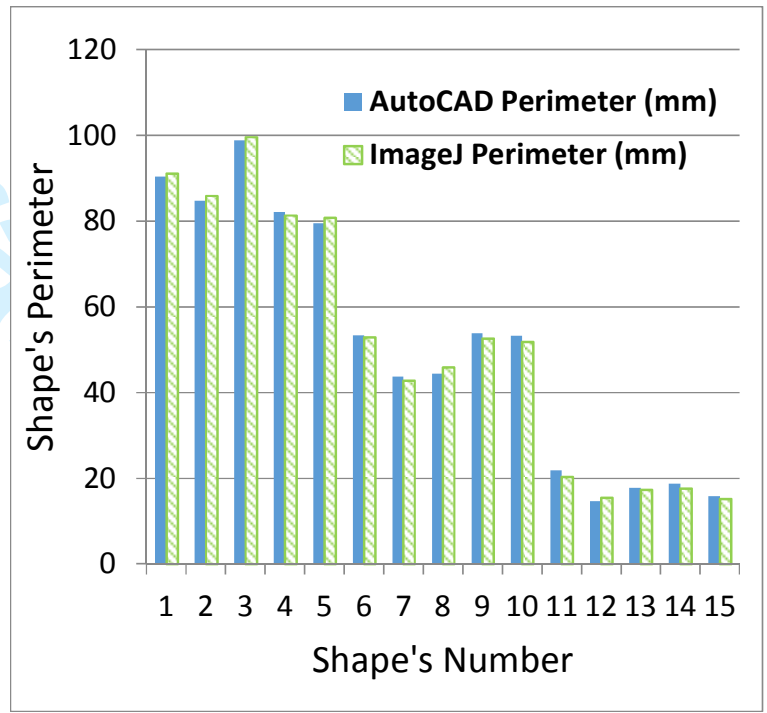

(d) Comparison between irregular shapes perimeters from AutoCAD and ImageJ.

Figure 7: Comparison between the collected data from AutoCAD with data from ImageJ analysis for the regular and irregular shapes. 


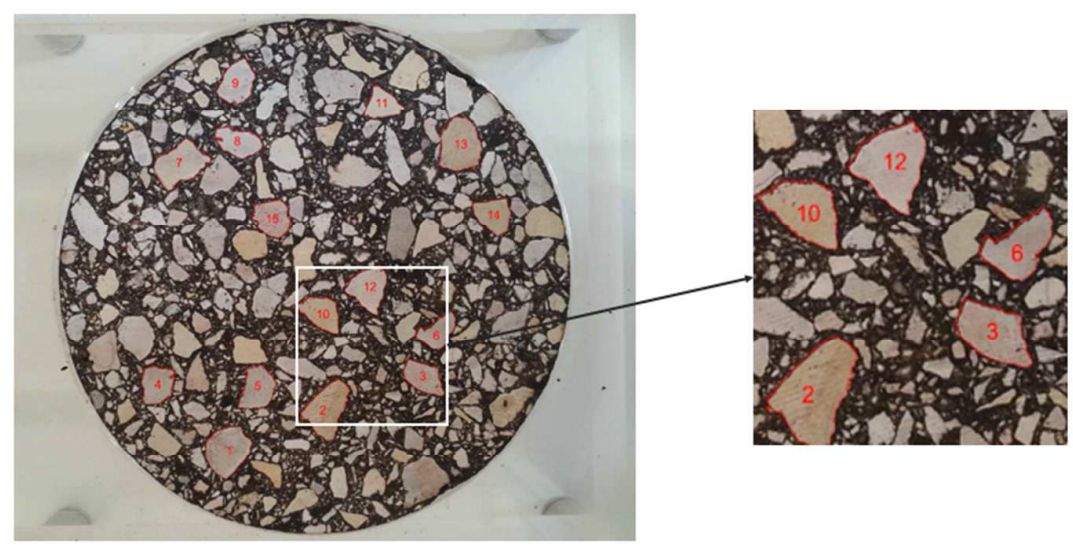

(a) Fifteen large particles.

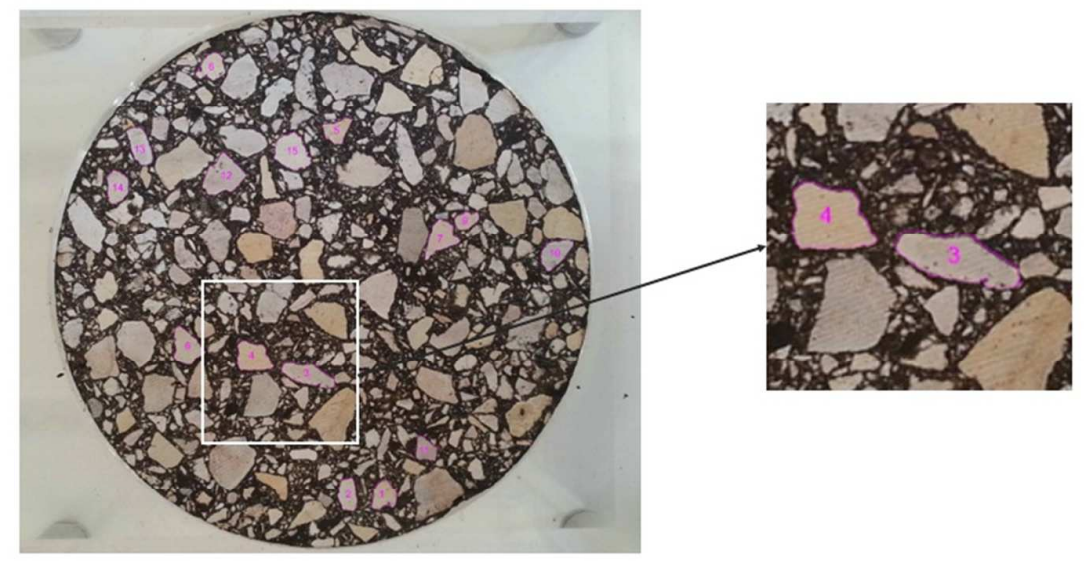

(b) Fifteen medium particles.

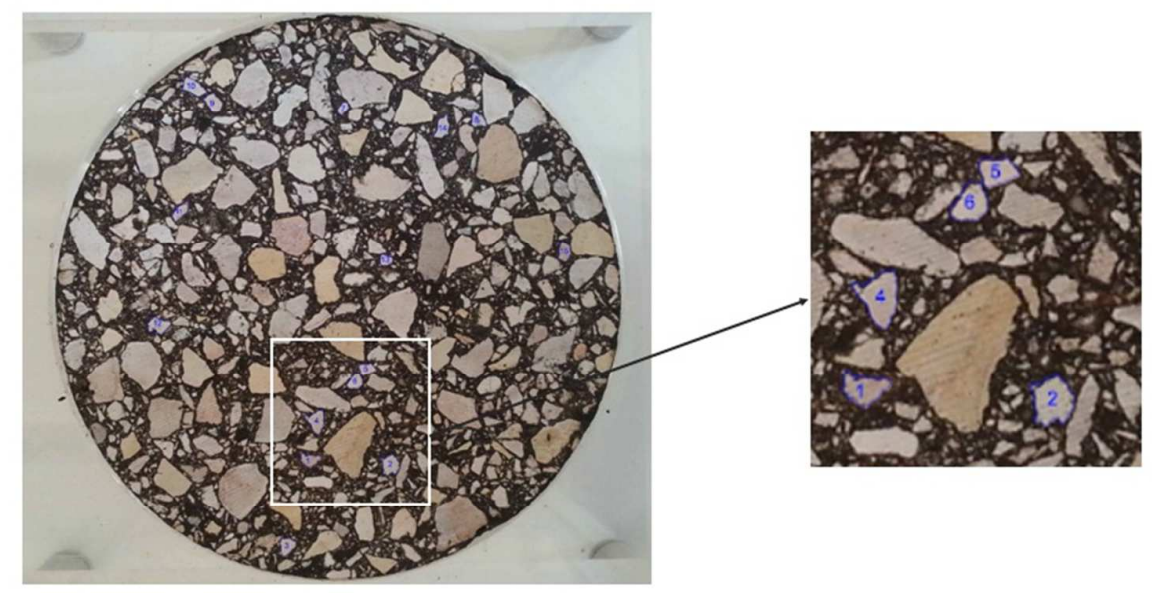

(c) Fifteen small particles.

Figure 8: The sizes' classification of the selected forty-five aggregate particles. 

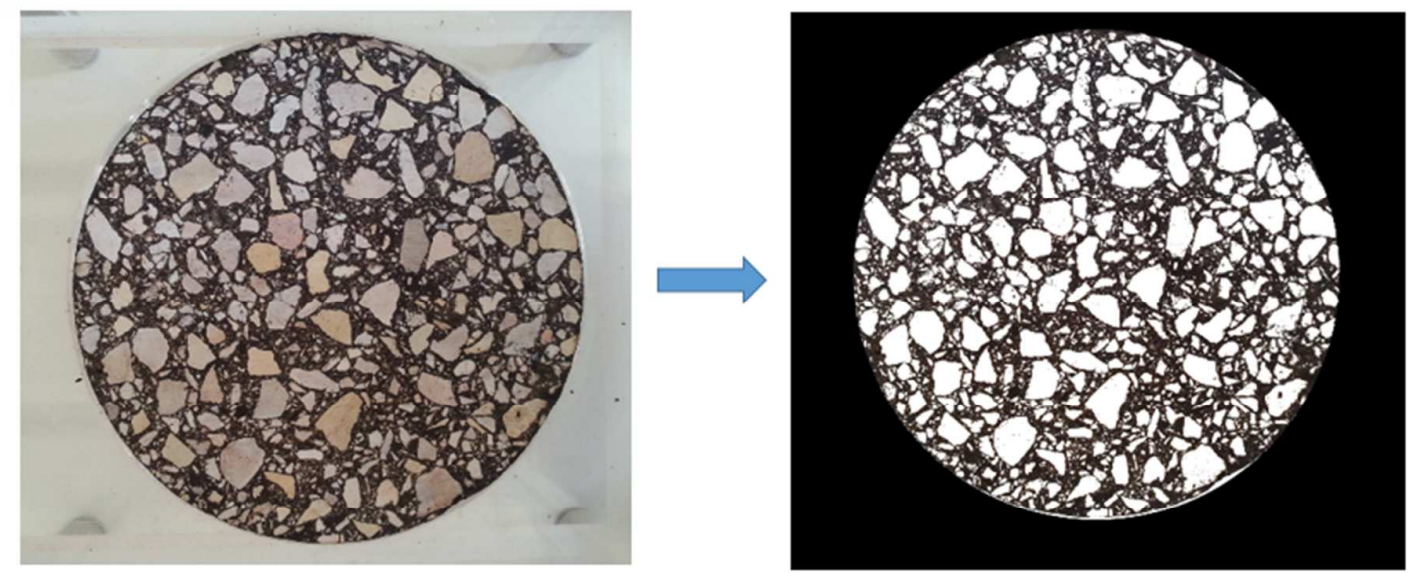

(a) Mixture slices face before and after Thresholding.

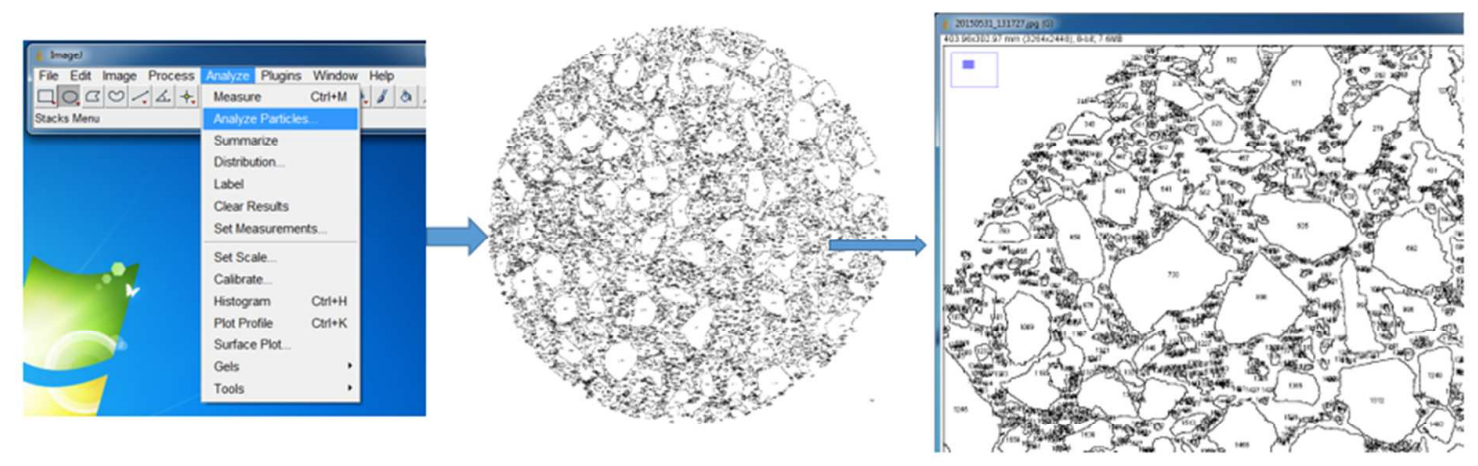

(b) Analysis of the particles, edge detection and particles labeling.

Figure 9: Thresholding and particle analysis. 


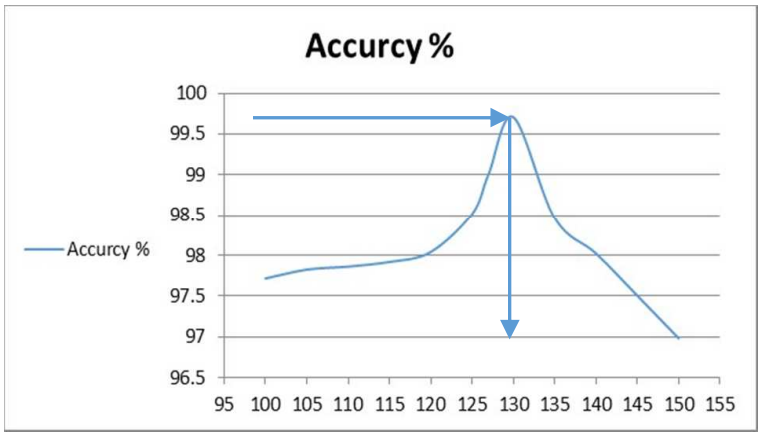

(a) The accuracy percentage according to the thresholds values for the large particles areas detetiction.

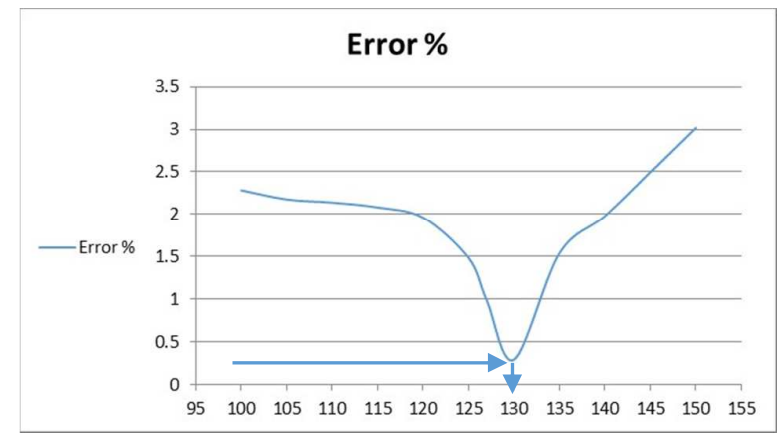

(b) The error percentage according to the thresholds values for the large particles areas detetiction.

Figure 10: An example for the percentage of accuracy and error for the computation of large particles areas by the Image J at the threshold values from 100 to 150 . 


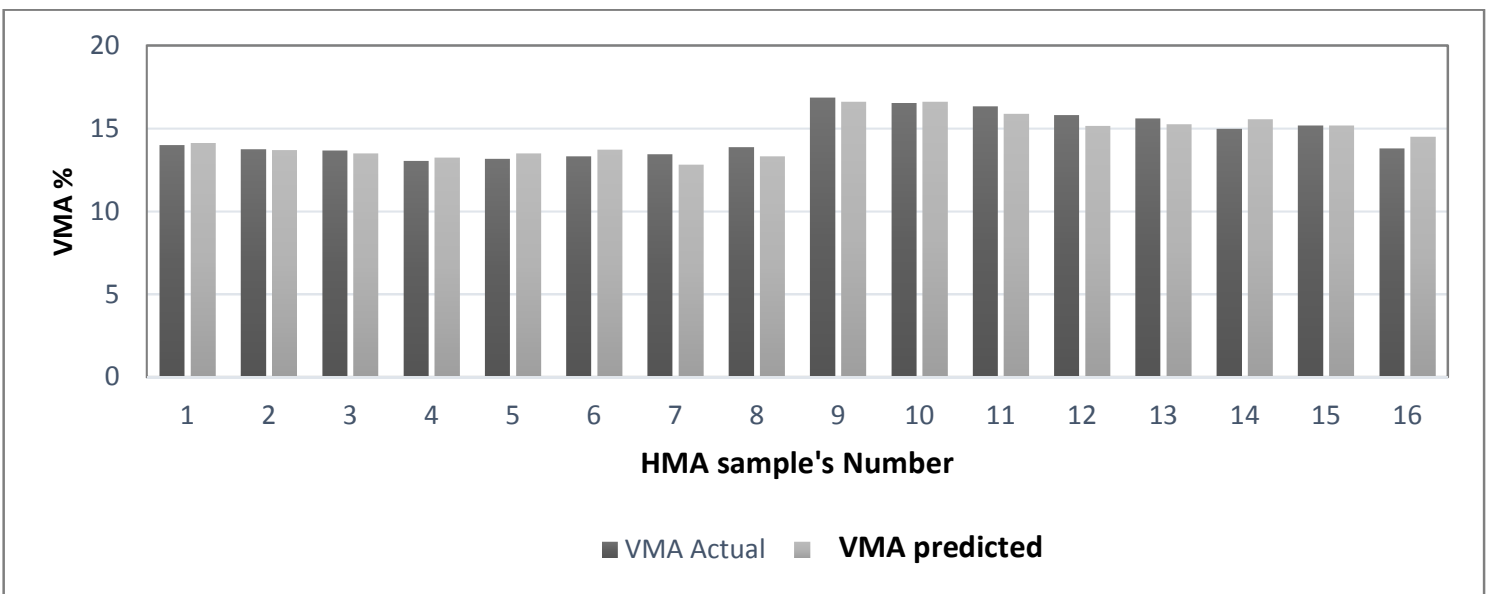

(a) Predicted values from the proposed compared with actual values of VMA.

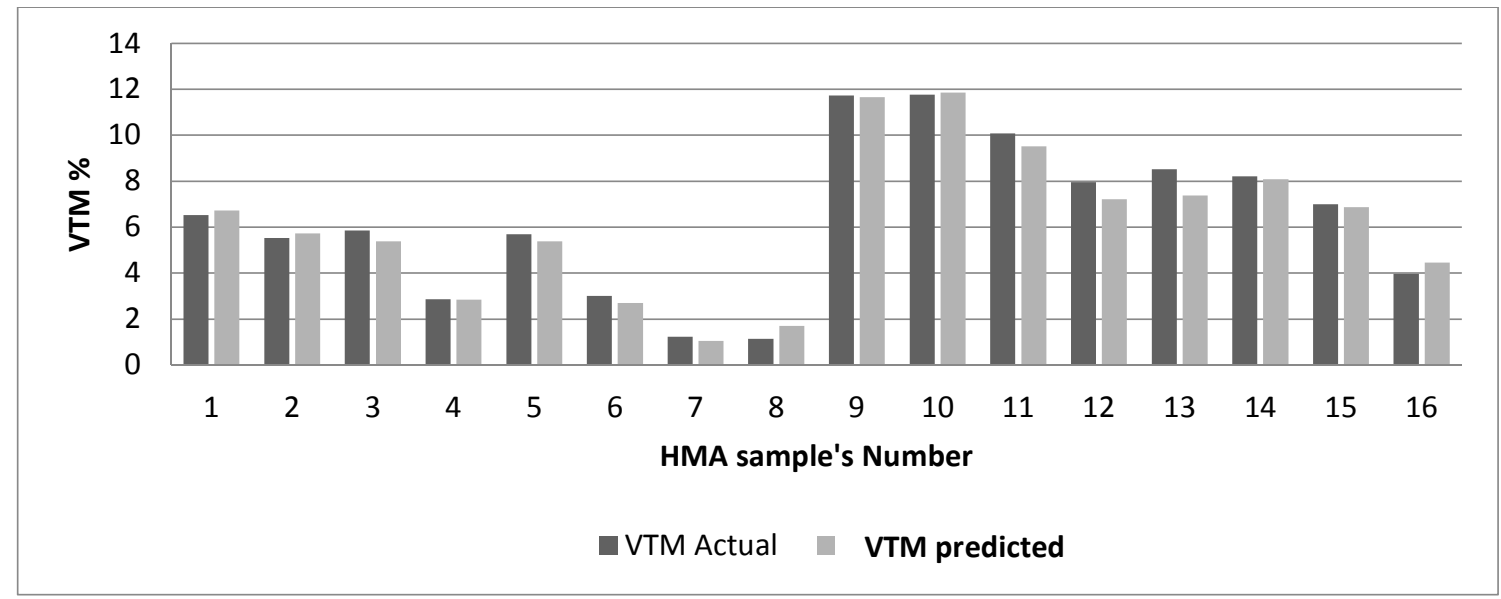

(b) Predicted values from the proposed model compared with actual values of VTM.

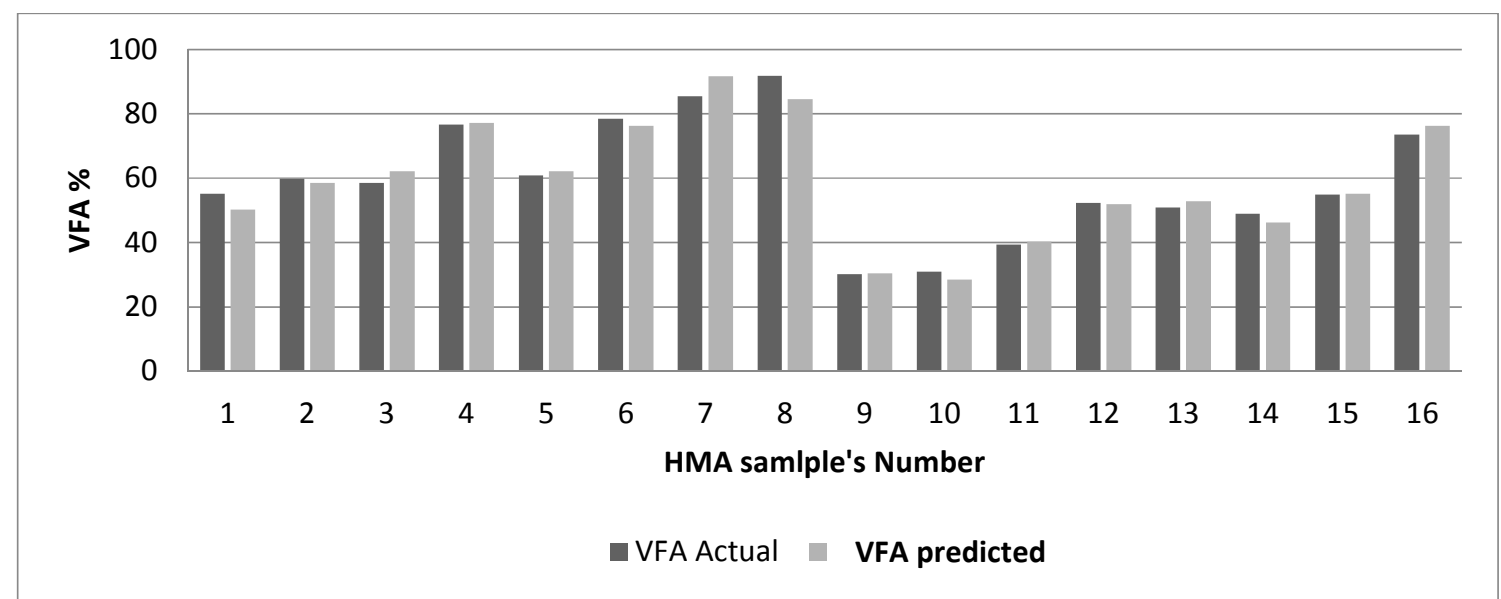

(c) Predicted values from the proposed model compared with actual values of VFA.

Figure 11: Comparison between the predicted value of VMA, VTM and VFA using the new models, respectively, and the actual value from the conventional methods 


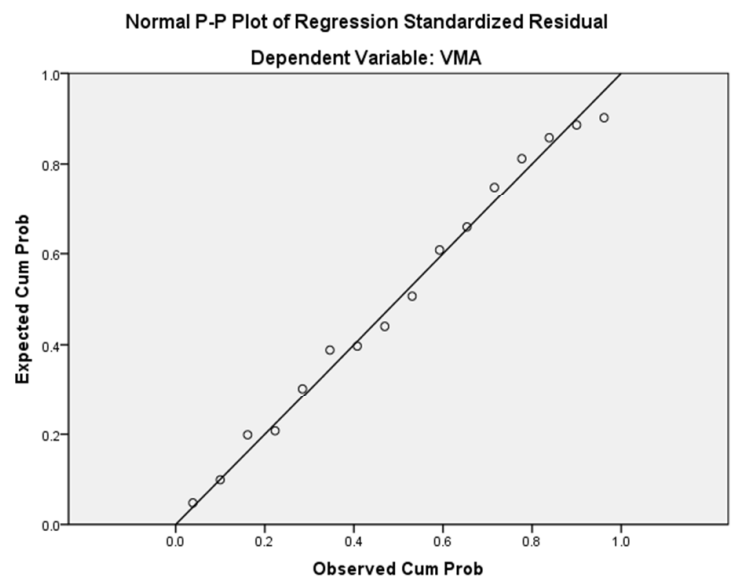

(a) Normal P-P plot of Reg. standardized Residual.

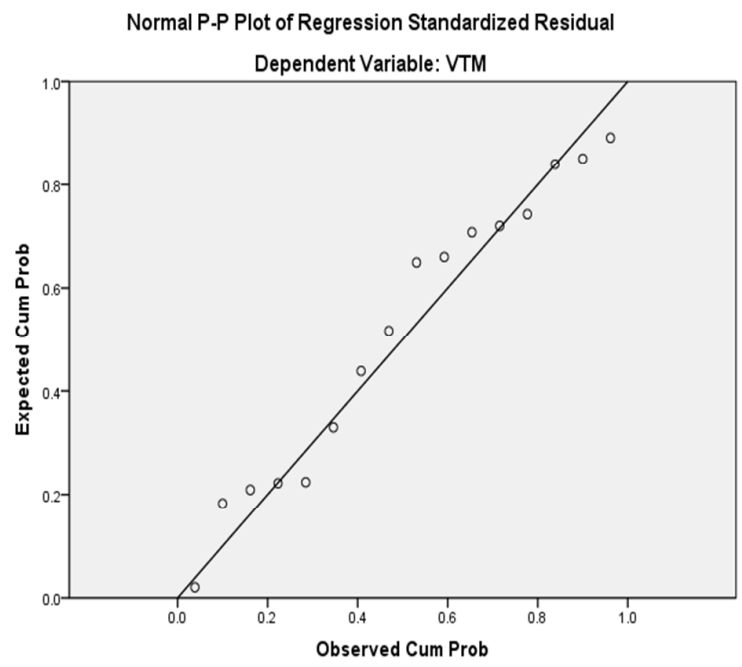

(c) Normal P-P plot of Reg. standardized Residual.

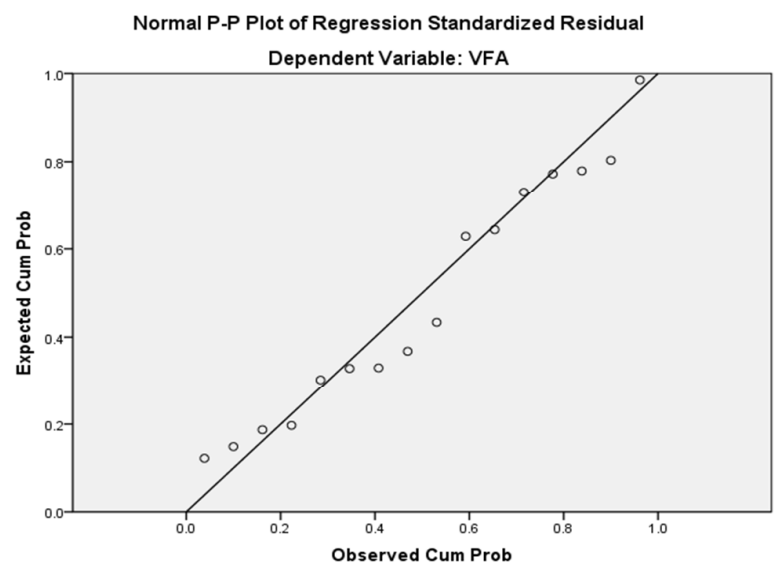

(e ) Normal P-P plot of Reg. standardized Residual.

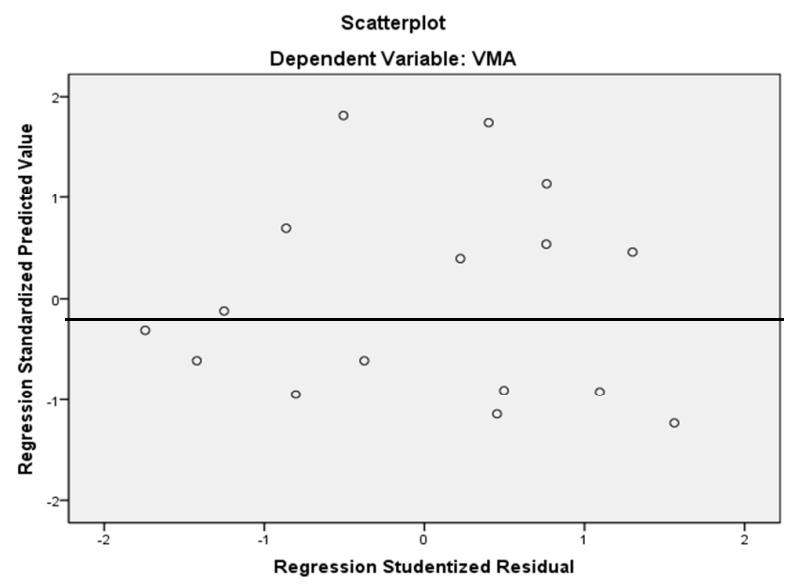

(b) Regression Predicted Value vs Regression Residual.

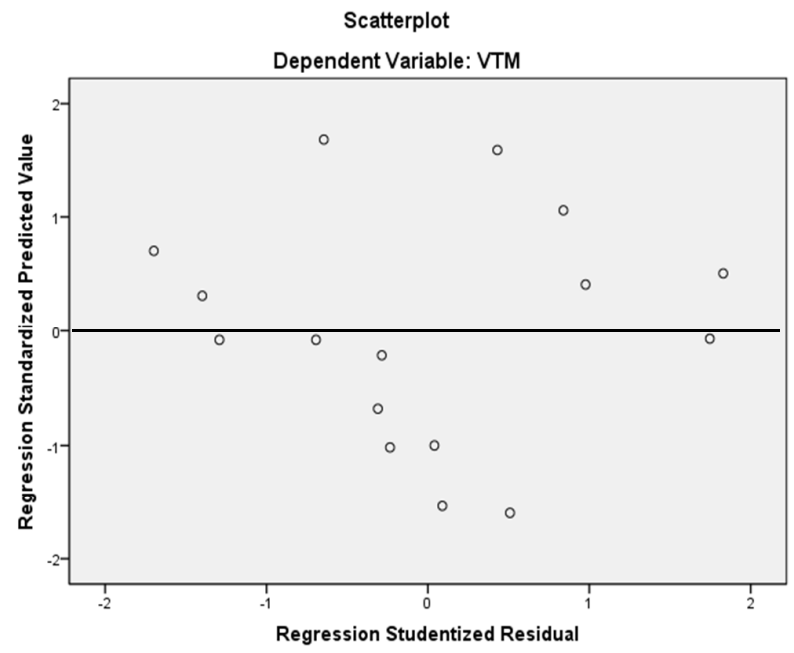

(d) Regression Predicted Value vs Regression Residual.

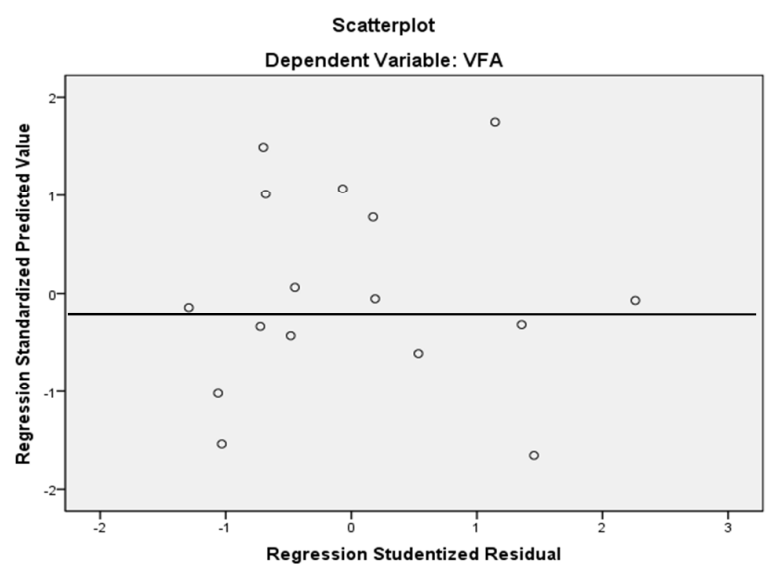

(f) Reg. Predicted Value Vs Regression Residual.

Figure 12: The linearity assumption checks for proposed models. 